\title{
lonized gas at the edge of the central molecular zone
}

\author{
W. D. Langer ${ }^{1}$, P. F. Goldsmith ${ }^{1}$ J. L. Pineda ${ }^{1}$, T. Velusamy ${ }^{1}$, M. A. Requena-Torres ${ }^{2}$, and H. Wiesemeyer ${ }^{2}$ \\ 1 Jet Propulsion Laboratory, California Institute of Technology, 4800 Oak Grove Drive, Pasadena, CA 91109, USA \\ e-mail: William.Langer@jpl.nasa.gov \\ 2 Max-Planck-Institut für Radioastronomie, Auf dem Hügel 69, 53121 Bonn, Germany
}

Received 18 November 2014 / Accepted 15 January 2015

\begin{abstract}
Context. The edge of the central molecular zone (CMZ) is the location where massive dense molecular clouds with large internal velocity dispersions transition to the surrounding more quiescent and lower $\mathrm{CO}$ emissivity region of the Galaxy. Little is known about the ionized gas surrounding the molecular clouds and in the transition region.

Aims. We determine the properties of the ionized gas at the edge of the CMZ near Sgr E using observations of $\mathrm{N}^{+}$and $\mathrm{C}^{+}$.

Methods. We observed a small portion of the edge of the CMZ near Sgr E with spectrally resolved [C II] $158 \mu \mathrm{m}$ and [N II] $205 \mu \mathrm{m}$ fine structure lines at six positions with the GREAT instrument on SOFIA and in [C II] using Herschel HIFI on-the-fly strip maps. We use the [N II] spectra along with a radiative transfer model to calculate the electron density of the gas and the [C II] maps to illuminate the morphology of the ionized gas and model the column density of CO-dark $\mathrm{H}_{2}$.

Results. We detect two [C II] and [N II] velocity components, one along the line of sight to a CO molecular cloud at $-207 \mathrm{~km} \mathrm{~s}^{-1}$ associated with Sgr E and the other at $-174 \mathrm{~km} \mathrm{~s}^{-1}$ outside the edge of another CO cloud. From the [N II] emission we find that the average electron density is in the range of $\sim 5$ to $21 \mathrm{~cm}^{-3}$ for these features. This electron density is much higher than that of the disk's warm ionized medium, but is consistent with densities determined for bright diffuse $\mathrm{H}$ II nebula. The column density of the CO-dark $\mathrm{H}_{2}$ layer in the $-207 \mathrm{~km} \mathrm{~s}^{-1}$ cloud is $\sim 1-2 \times 10^{21} \mathrm{~cm}^{-2}$ in agreement with theoretical models. The CMZ extends further out in Galactic radius by $\sim 7$ to $14 \mathrm{pc}$ in ionized gas than it does in molecular gas traced by $\mathrm{CO}$.

Conclusions. The edge of the CMZ likely contains dense hot ionized gas surrounding the neutral molecular material. The high fractional abundance of $\mathrm{N}^{+}$and high electron density require an intense EUV field with a photon flux of order $10^{6}$ to $10^{7}$ photons $\mathrm{cm}^{-2} \mathrm{~s}^{-1}$, and/or efficient proton charge exchange with nitrogen, at temperatures of order $10^{4} \mathrm{~K}$, and/or a large flux of X-rays. Sgr $\mathrm{E}$ is a region of massive star formation as indicated by the presence of numerous compact $\mathrm{H}$ II regions. The massive stars are potential sources of the EUV radiation that ionizes and heat the gas. In addition, X-ray sources and the diffuse X-ray emission in the CMZ are candidates for ionizing nitrogen.
\end{abstract}

Key words. ISM: clouds - HII regions - Galaxy: center

\section{Introduction}

The central molecular zone (CMZ), the central $\sim 400$ pc by $\sim 80 \mathrm{pc}$ of the Galaxy, is a region filled with massive dense giant molecular clouds (GMC) having large internal velocity dispersion. Characteristic cloud line widths are $\geq 20 \mathrm{~km} \mathrm{~s}^{-1}$, compared to a few $\mathrm{km} \mathrm{s}^{-1}$ for GMCs in the disk (e.g. Morris \& Serabyn 1996; Oka et al. 1998; Ferrière et al. 2007). In addition, GMCs in the CMZ have higher kinetic temperatures, $T_{k} \sim 30$ to $200 \mathrm{~K}$ (Ao et al. 2013; Mills \& Morris 2013), and densities, $n\left(\mathrm{H}_{2}\right) \geq$ $10^{4-5} \mathrm{~cm}^{-3}$ (e.g. Jackson et al. 1996; Oka et al. 1998; Dame et al. 2001; Martin et al. 2004), than GMCs in the disk. The gas distribution within the inner Galaxy and across the edge of the CMZ is thought to be due to the gravitational potential and dynamics of the gas there (see overview by Ferrière et al. 2007). While the atomic and molecular hydrogen distributions in the CMZ have been mapped with spectrally resolved $\mathrm{HI}$ and $\mathrm{CO}$, respectively, less is known about the distribution and properties of the ionized gas in this region.

The electron distribution of the interstellar medium has been determined from dispersion measurements of radio emission from pulsars and other compact radio sources (Cordes \& Lazio 2002, 2003). Lazio \& Cordes (1998) and Roy (2013) find that the average electron density in the inner $100 \mathrm{pc}$ is $\langle n(\mathrm{e})\rangle \sim 10 \mathrm{~cm}^{-3}$.
The radial distribution has been modeled as a Gaussian with scale length of $145 \mathrm{pc}$ (see Ferrière et al. 2007), which yields a value at the edge of the CMZ $\langle n(\mathrm{e})\rangle \sim 1-2 \mathrm{~cm}^{-3}$. Lazio \& Cordes (1998) proposed that the scattering of radio waves is not due to a uniform electron distribution but occurs in thin ionized layers at the surface of molecular clouds with $n(\mathrm{e}) \gtrsim 10^{3} \mathrm{~cm}^{-3}$ and $T_{k}(\mathrm{e}) \sim 10^{3} \mathrm{~K}$, or at the interface with the hot ionized medium (HIM) with $n(\mathrm{e}) \sim 5-50 \mathrm{~cm}^{-3}$ and $T_{k}(\mathrm{e}) \sim 10^{5}-10^{6} \mathrm{~K}$. Observations of the spectrally resolved emission from ions in the CMZ can help distinguish among these different models and provide information about the density, filling factor, and ionization mechanisms. The objective of this paper is to explore the nature of the ionized gas at the edge of the CMZ near Sgr E using spectrally resolved emission from the fine structure lines, $[\mathrm{N}$ II] and [C II $]$, of ionized nitrogen, $\mathrm{N}^{+}$, and ionized carbon, $\mathrm{C}^{+}$, respectively.

The Balloon-borne Infrared Carbon Explorer (BICE) mapped $\left[\mathrm{C}_{\mathrm{II}}\right]$ in the inner Galaxy with modest spatial $\left(15^{\prime}\right)$ and low spectral $\left(\sim 175 \mathrm{~km} \mathrm{~s}^{-1}\right)$ resolution (Nakagawa et al. 1998). BICE detected bright $[\mathrm{C}$ II] emission from the CMZ from $358.3<l<1^{\circ}$ but tapering off outside, and confined to $|b| \lesssim 1^{\circ}$ (see their Figs. 7 and 8). Within the limitations of BICE's low spectral resolution, Nakagawa et al. (1998) found that $[\mathrm{CII}]$ has a large velocity dispersion towards the CMZ, 
$\Delta v \geq 100 \mathrm{~km} \mathrm{~s}^{-1}$ (see their Fig. 14), similar to the dispersion seen in ${ }^{12} \mathrm{CO}$. In addition, the Infrared Space Observatory (ISO) observed [C II] and [N II] along a number of lines of sight in the CMZ (see Rodríguez-Fernández et al. 2004) with a spectral resolution $\sim 30 \mathrm{~km} \mathrm{~s}^{-1}$. However, much better spectral resolution is needed to separate individual components and determine gas characteristics.

To understand better the distribution of the ionized gas at the edge of the CMZ we observed several lines of sight in [N II] and [C II] in July 2013 using the heterodyne German REceiver for Astronomy at Terahertz frequencies (GREAT ${ }^{1}$; Heyminck et al. 2012) onboard the Stratospheric Observatory for Infrared Astronomy (SOFIA; Young et al. 2012). In addition, prior to the GREAT observations, we made small scale strip maps in [C II] to map the overall distribution of ionized gas as part of a Herschel ${ }^{2}$ (Pilbratt et al. 2010) Open Time 2 Programme, using the high spectral resolution Heterodyne Instrument in the Far Infrared (HIFI; de Graauw et al. 2010).

Here we report on the results of these observations, which indicate that there are two regions of highly ionized, high temperature, dense gas: one along the line of sight that includes a $\mathrm{CO}$ molecular cloud associated with $\mathrm{Sgr} \mathrm{E}$ at $V_{\mathrm{lsr}} \sim-207 \mathrm{~km} \mathrm{~s}^{-1}$ and the other at $V_{\mathrm{lsr}} \sim-174 \mathrm{~km} \mathrm{~s}^{-1}$ outside the edge of a CO molecular cloud located at larger $l$ in the CMZ. The electron densities derived from the [N II] emission, $n(\mathrm{e}) \sim 5$ to $25 \mathrm{~cm}^{-3}$, are two to three orders of magnitude higher than that in the disk's warm ionized medium (WIM), but consistent with those found from analysis of [N II] emission in the Carina nebula, a luminous HII region with numerous O stars (Oberst et al. 2011). This paper is organized as follows. In Sect. 2 we present our observations, while Sect. 3 analyzes the distribution and density of the ionized gas. Section 4 discusses the possible ionization sources, and Sect. 5 summarizes the results.

\section{Observations of [ $\mathrm{C} I I]$ and $[\mathrm{NII}]$}

In Fig. 1 we show a portion of the NANTEN 4-m CO map from Fig. 1a in Fukui et al. (2006), and reprinted with permission from AAAS, that zooms in on the CO edge of the CMZ. The yellow box in Fig. 1 outlines the region mapped with HIFI, and the positions observed in [C II] and [N II] with GREAT are indicated by red crosses. [N II] traces just the highly ionized gas, while [C II] traces highly ionized gas and the weakly ionized neutral atomic and molecular gas. Located just inside the edge of the $\mathrm{CMZ}$ is Sgr E, a region with many radio sources located around $l \sim 358^{\circ} .7$ and $b \sim 0^{\circ}$ (Gray 1994), which contains 18 compact $\left(<2^{\prime}\right)$ H II regions and diffuse emission (Liszt 1992). Sgr E is a region of active star formation associated with a $\mathrm{CO}$ molecular cloud with $V_{\mathrm{lsr}} \sim-210 \mathrm{~km} \mathrm{~s}^{-1}$ (Oka et al. 1998). The radio recombination line survey, H II Region Discovery Survey (HRDS: Anderson et al. 2011, 2012) detected eight of these H II sources towards Sgr E at $V_{\mathrm{lsr}} \sim-208 \mathrm{~km} \mathrm{~s}^{-1}$, the same velocity as the $\mathrm{CO}$ cloud. Along the line of sight outside the CMZ there are giant magnetic loops, large scale molecular gas loop-like features, with large line widths and large scale flows detected in CO (Fukui et al. 2006; Fujishita et al. 2009). The edge of Loop 1 intercepts the plane at $l \sim 358^{\circ} .2$ to $358^{\circ} .4$, close to the line of

\footnotetext{
GREAT is a development by the MPI für Radioastronomie and KOSMA/Universität $\mathrm{zu} \mathrm{Köln,} \mathrm{in} \mathrm{cooperation} \mathrm{with} \mathrm{the} \mathrm{MPI} \mathrm{für}$ Sonnensystemforschung and the DLR Institut für Planentenforschung.

2 Herschel is an ESA space observatory with science instruments provided by European-led Principal Investigator consortia and with important participation from NASA.
}

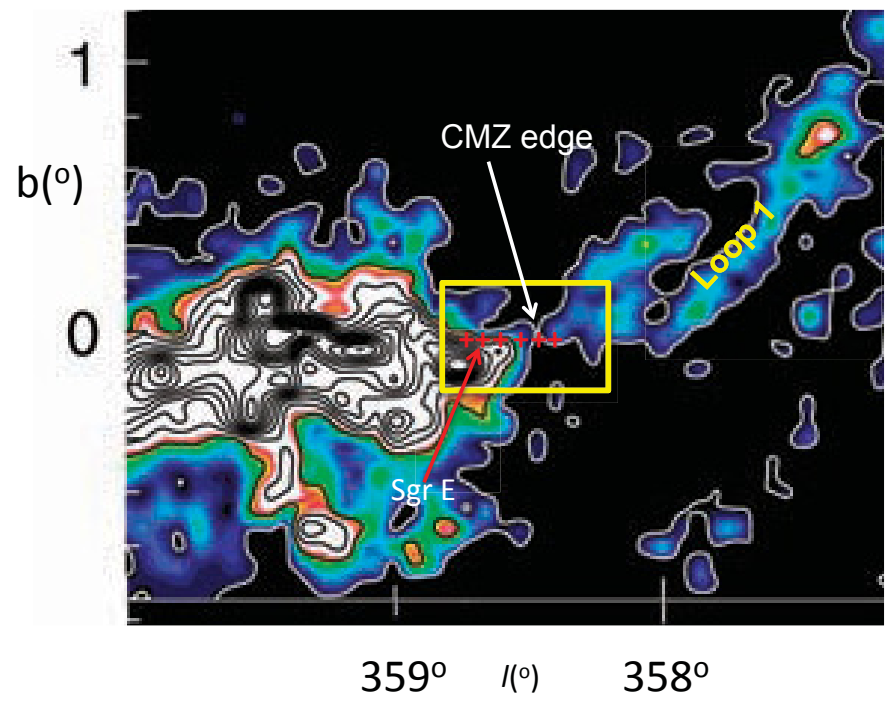

Fig. 1. A portion of a map of CO integrated intensity from -180 to $-90 \mathrm{~km} \mathrm{~s}^{-1}$ of the edge of the CMZ from Fukui et al. (2006), and reprinted with permission from AAAS, based on the NANTEN $\mathrm{CO}(1-0)$ map which has a beam size $\sim 3^{\prime}$. The region mapped with HIFI is outlined by the yellow box and the positions of the [C II] and [N II] observations with GREAT are indicated by crosses. The approximate $\mathrm{CO}$ edge of the $\mathrm{CMZ}$ and a portion of Loop 1 are indicated, as is the location of Sgr E.

sight to the edge of the CMZ, and has a $V_{\text {lsr }} \sim-135 \mathrm{~km} \mathrm{~s}^{-1}$ (Fukui et al. 2006). This loop, however, is at a Galactic radius, $R_{\mathrm{G}} \sim 670$ pc (Torii et al. 2010), well outside the CMZ.

\subsection{SOFIA [CII and [N II] data}

We observed the lower fine structure transition of ionized nitrogen, [N II] ${ }^{3} \mathrm{P}_{1} \rightarrow{ }^{3} \mathrm{P}_{0}$ at $1461.1319 \mathrm{GHz}(205.2 \mu \mathrm{m})$ and, simultaneously, the fine structure line of ionized carbon, [C II] ${ }^{2} \mathrm{P}_{3 / 2} \rightarrow{ }^{2} \mathrm{P}_{1 / 2}$ at $1900.5369 \mathrm{GHz}(157.7 \mu \mathrm{m})$, using GREAT. The angular resolution is $15^{\prime \prime}$ at $[\mathrm{C} \mathrm{II}]$ and $20^{\prime \prime}$ at [N II]. To improve the signal-to-noise ratio $(\mathrm{S} / \mathrm{N})$ the $[\mathrm{C}$ II $]$ and $[\mathrm{N}$ II $]$ data were smoothed to a velocity resolution of $1.20 \mathrm{~km} \mathrm{~s}^{-1}$ and $1.57 \mathrm{~km} \mathrm{~s}^{-1}$ per channel, respectively. The spectra were calibrated using the procedure outlined in Guan et al. (2012).

The GREAT [C II] observations contained atmospheric features in the spectral region observed due to the frequency setting for large negative velocities of the source, and the [C II] line was in the wing of a strong water line. Moreover, the position switching observing mode made the presence of this feature a significant issue that needed to be addressed in the data reduction. The standard atmospheric calibration was not sufficient to produce a satisfactory fit to the water feature, and we had to use a set of non-standard parameters in the atmospheric model (Guan et al. 2012) to fit the width and the peak of the water line. The main change to the normal procedure was to reduce the contribution from the Dry atmosphere in steps, allowing the Wet part (water) to fit the atmospheric feature. We implemented a set of six models, and chose the model that best fit the water feature. This feature was removed and a zero order spectral baseline fit applied to the delivered data products. The resulting spectra were, with one exception, fairly regular with residual low order polynomial variations and only slight offsets from zero; one spectrum had a long period, but well ordered, variation in the baseline. In order to fit the baselines better than zero order, the delivered data 


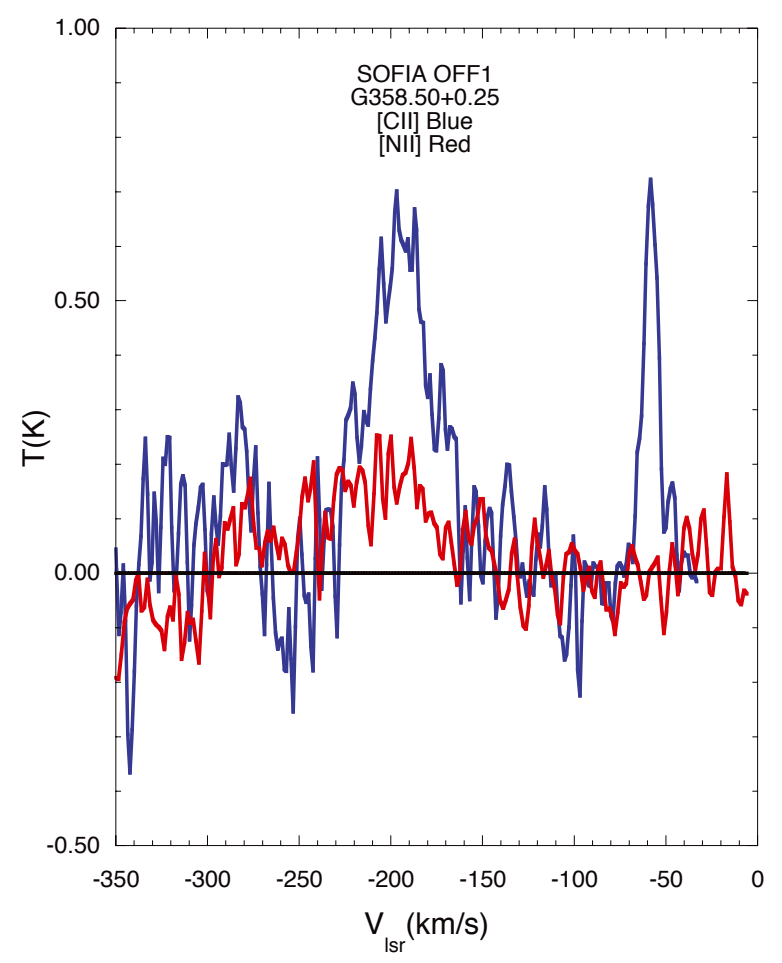

Fig. 2. $[\mathrm{C}$ II $]$ and $[\mathrm{N}$ II $]$ emission in the reference OFF1 position at $l=$ $358^{\circ} .50$ and $b=0.25$. The data have been smoothed to $2.4 \mathrm{~km} \mathrm{~s}^{-1}$ for $\left[\mathrm{C}\right.$ II] and $3.1 \mathrm{~km} \mathrm{~s}^{-1}$ for $[\mathrm{N}$ II] to improve the $\mathrm{S} / \mathrm{N}$. The [C II] emission peaks at $V_{\mathrm{lsr}} \sim-195 \mathrm{~km} \mathrm{~s}^{-1}$ while that from [N II] peaks $\sim-207 \mathrm{~km} \mathrm{~s}^{-1}$, within the fitting errors of the weaker [N II] signal. The narrow [C II] emission at $V_{\mathrm{lsr}} \sim-58 \mathrm{~km} \mathrm{~s}^{-1}$ arises from the $3 \mathrm{kpc}$ arm. [N II] is not detected from this arm.

were then reduced further in $\mathrm{CLASS}^{3}$ and baselines corrected with a polynomial fitting routine. Comparison of the GREAT and HIFI [C II] data at one nearly coincident position (see below) shows excellent agreement, lending confidence to the removal of the water line and overall baseline fittings. The intensities were corrected for a main beam efficiency of 0.67 . The rms noise for the final $[\mathrm{C} \mathrm{II}]$ products ranges from 0.16 to $0.26 \mathrm{~K}$ per $1.20 \mathrm{~km} \mathrm{~s}^{-1}$ channel, while that for [N II] ranges from 0.09 to $0.23 \mathrm{~K}$ per $1.57 \mathrm{~km} \mathrm{~s}^{-1}$ channel.

We observed six lines of sight (LOS) along $b=0^{\circ}$ at longitudes from $l=358^{\circ} .45$ to $358^{\circ} 75$. The reference off-position, OFF $1, l=358.50$ and $b=0.25$ contains weak [C II] and very weak [N II] emission, shown in Fig. 2, so that all the onpositions had to be corrected for this emission. To correct the observations for emission in OFF1 it was observed separately on two nights as an on-position using a different off-position, OFF2, $l=358.50$ and $b=0.65$. The OFF1 lines appear to be singly peaked and a Gaussian fit to the $[\mathrm{C}$ II] emission in OFF1 peaks at $V_{\text {lsr }} \sim-195 \mathrm{~km} \mathrm{~s}^{-1}$ and has a full width half maximum $(\mathrm{FWHM}) \sim 43 \mathrm{~km} \mathrm{~s}^{-1}$. The narrow [C II] feature at $V_{\text {lsr }} \sim-58 \mathrm{~km} \mathrm{~s}^{-1}\left(F W H M \sim 9 \mathrm{~km} \mathrm{~s}^{-1}\right)$ arises from the $3 \mathrm{kpc}$ arm. A Gaussian fit to the broad, weak [N II] line yields a peak $V_{\mathrm{lsr}} \sim-205 \mathrm{~km} \mathrm{~s}^{-1}$ and a $F W H M \sim 65 \mathrm{~km} \mathrm{~s}^{-1}$, close to the characteristics of the [C II] line considering the weakness of the line and the low $\mathrm{S} / \mathrm{N}$ of this detection. No other $[\mathrm{N} \mathrm{III}$ is detected in the band above the $3 \sigma$ level.

As a check on the quality of the GREAT data, given that it had to be corrected for the atmospheric water line and emission

\footnotetext{
3 http://wWw.iram.fr/IRAMFR/GILDAS
}

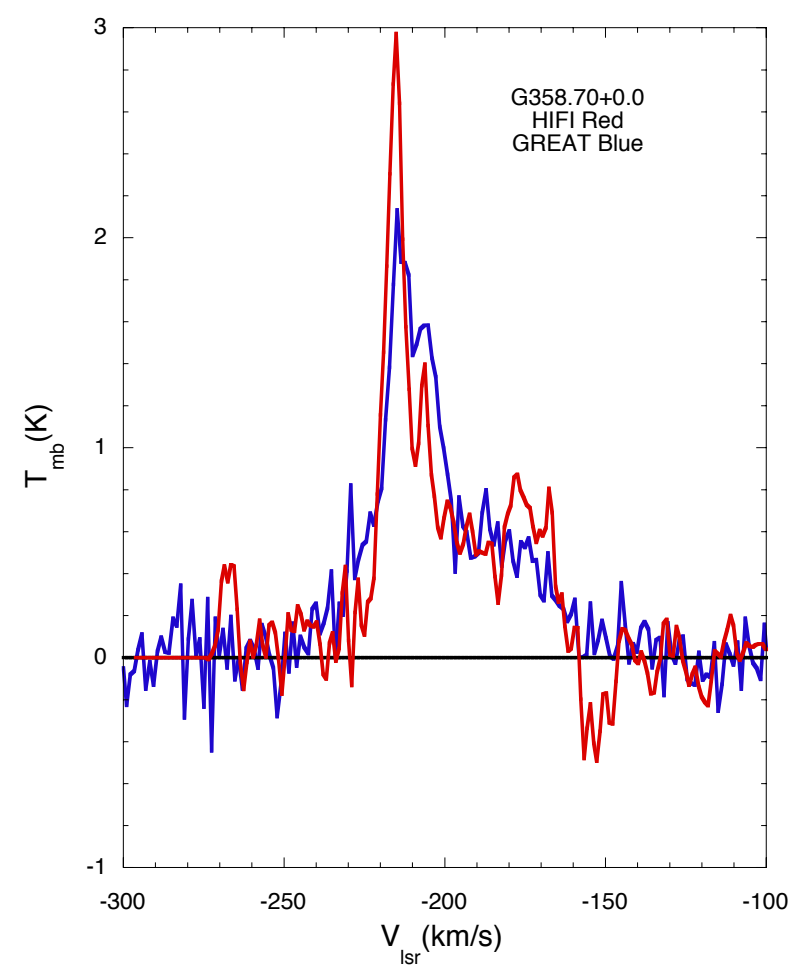

Fig. 3. GREAT [C II] spectrum at the position observed with GREAT, $l=358^{\circ} .700$ and $b=0.0$, that is closest to a $[\mathrm{C} \mathrm{II}]$ spectrum from GOT $\mathrm{C}+$. The GOT C+ position $l=358.696$ and $b=0.0$ is offset in longitude by $14^{\prime \prime}$ from the GREAT pointing, which is about the GREAT beam width. The HIFI data have been smoothed to the resolution of the GREAT data, $1.2 \mathrm{~km} \mathrm{~s}^{-1}$. The [C II] GREAT spectrum is similar to that from HIFI showing both the $-207 \mathrm{~km} \mathrm{~s}^{-1}$ and $-174 \mathrm{~km} \mathrm{~s}^{-1}$ components. The total integrated intensities agree within $\pm 10 \%$.

in the OFF1 position, we show in Fig. 3 a spectrum from the HIFI Galactic Observations of Terahertz (GOT C+) [C II] survey (Langer et al. 2010; Pineda et al. 2013) at the closest position, $l=358.696$ and $b=0.0$, to the GREAT observation at $l=358^{\circ} 700$ and $b=0.0$. The offset in longitude of $14^{\prime \prime}$ corresponds to 1.2 HIFI and 0.9 GREAT beam widths, and some portions of the main beams are only about an arcsecond apart. The [C II] emission from HIFI and GREAT have the same distribution in velocity, both show a strong peak at $\sim-210 \mathrm{~km} \mathrm{~s}^{-1}$ with intensities that generally agree within the uncertainties of the baseline fitting, and both show weak emission centered around $\sim-175 \mathrm{~km} \mathrm{~s}^{-1}$ that blends into the stronger feature. The integrated intensities of these two observations over the velocity range $-150 \mathrm{~km} \mathrm{~s}^{-1}$ to $-250 \mathrm{~km} \mathrm{~s}^{-1}$ differ by only $10 \%$. Furthermore, the GOT C+ spectrum used a different off position than the GREAT observations, lending further support for our procedure to correct the GREAT [C II] and [N II] for emission in the GREAT OFF1 position.

The [C II] and [N II] spectra along $b=0^{\circ}$ are shown in Fig. 4. [C II] emission was detected at all six positions and there are two adjacent, and at times blended, features arising from the CMZ in the velocity range $\sim-150 \mathrm{~km} \mathrm{~s}^{-1}$ to $-250 \mathrm{~km} \mathrm{~s}^{-1}$. At the two positions where these components are clearly separated, the peaks occur at $V_{\mathrm{lsr}} \sim-174$ and $-207 \mathrm{~km} \mathrm{~s}^{-1}$. In general the $-174 \mathrm{~km} \mathrm{~s}^{-1}$ component is weaker than the $-207 \mathrm{~km} \mathrm{~s}^{-1}$ component, and both features are broad with a typical FWHM of $\sim 25 \mathrm{~km} \mathrm{~s}^{-1}$. No emission is detected at $V_{\mathrm{lsr}} \sim-135 \mathrm{~km} \mathrm{~s}^{-1}$, the velocity of Loop 1. 

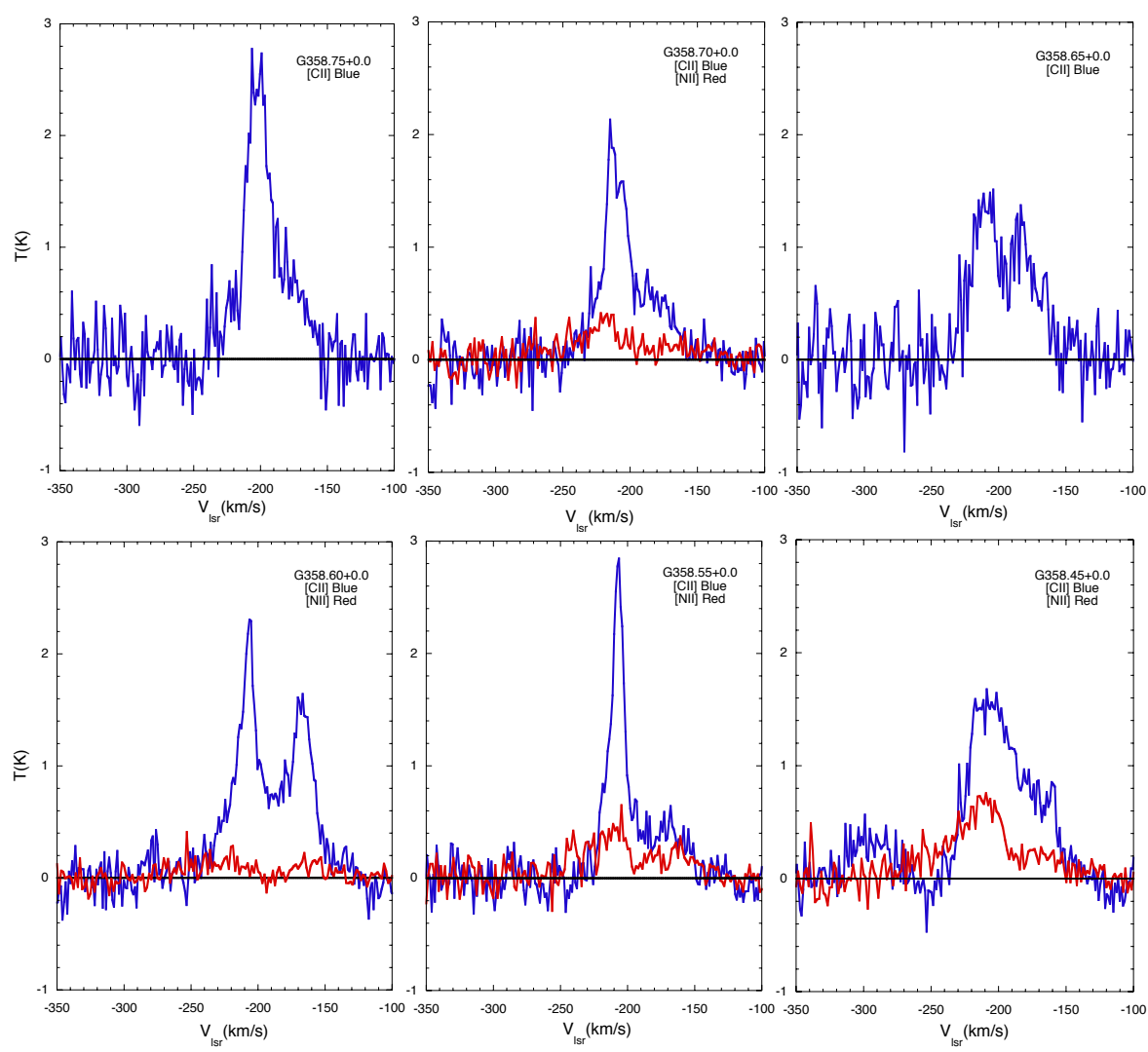

Fig. 4. [C II] (blue) and [N II] (red) spectra at the six positions observed with GREAT along $b=0.0$. The Galactic longitude and latitude are indicated by GXXX.XX+Y.Y. The two features centered at $\sim-174 \mathrm{~km} \mathrm{~s}^{-1}$ and $\sim-207 \mathrm{~km} \mathrm{~s}^{-1}$ (which are blended at some positions) arise from the edge of the CMZ. [C II] is detected at all 6 positions in the $\mathrm{CMZ}$, but $[\mathrm{N} \mathrm{II}]$ is only detected at 4 positions. The two non-detections in [N II] are associated with greater rms noise. While [N II] is generally much weaker than $[\mathrm{CII}]$, the $[\mathrm{N}$ II] peak temperature at $l=358^{\circ} .45$ is about half that of [C II].
[N II] is only detected at four of the six LOS, and is generally much weaker than $[\mathrm{C}$ II $]$ except at $l=358^{\circ} .45$ where the component at $V_{\mathrm{lsr}} \sim-207 \mathrm{~km} \mathrm{~s}^{-1}$ is about half as intense as [C II]. The four LOS where $[\mathrm{N} \mathrm{II}]$ is detected have an rms noise of $\sim 0.1 \mathrm{~K}$ per $1.57 \mathrm{~km} \mathrm{~s}^{-1}$ channel whereas the two lines of sight where $[\mathrm{N} \mathrm{II}]$ is not detected have a larger rms noise, $\sim 0.23 \mathrm{~K}$. The [N II] components were fit with a double Gaussian with one peak at $\sim-215 \mathrm{~km} \mathrm{~s}^{-1}$ and a $F W H M \sim 35 \mathrm{~km} \mathrm{~s}^{-1}$ and the other component is at $\sim-170 \mathrm{~km} \mathrm{~s}^{-1}$ with a $F W H M \sim 25 \mathrm{~km} \mathrm{~s}^{-1}$. All the [C II] features have a large $\mathrm{S} / \mathrm{N}$ while the $[\mathrm{N}$ II] $\mathrm{S} / \mathrm{N}$ ranges from $\sim 4$ to 20 for the four LOS in which the line was detected.

\subsection{Herschel [CII] map data}

The GREAT observations provide [C II] at six and [N II] at four longitudinal positions in the plane $\left(b=0^{\circ}\right)$. To get insight into the distribution of [C II] above and below the plane we use [C II] maps made with the high spectral resolution HIFI (de Graauw et al. 2010) instrument onboard Herschel (Pilbratt et al. 2010). We used a number of on-the-fly (OTF) scans to map the $0.6 \times$ 0.4 region shown in Fig. 1. All HIFI OTF scans were made in the LOAD-CHOP mode using a reference off-source position at $l=358^{\circ} .80, b=1.90$, about 1.7 away in latitude from the top of the map. The map consists of 13 scans in Galactic latitude, centered at $b=0^{\circ}$ and stepping in longitude at $3^{\prime}(0.05)$ intervals from $358^{\circ} .20$ to $358^{\circ} 80$. The OTF $b$-scans are $24^{\prime}$ long, sampled at every $40 "$. At $1.9 \mathrm{THz}$ the angular resolution of the Herschel telescope is $12^{\prime \prime}$, corresponding to a spatial resolution of $\sim 0.5 \mathrm{pc}$ at $8.5 \mathrm{kpc}$, the distance to the Galactic center. However the effects of scanning rate and the $40^{\prime \prime}$ sampling of the OTF maps degrades the effective resolution in $b$ to $80^{\prime \prime}$ while it is $12^{\prime \prime}$ in $l$. Thus spatial structures on scale sizes smaller than $80^{\prime \prime}$ in $b$ will be strongly attenuated. Furthermore the total duration of each OTF scan was typically $\sim 2000 \mathrm{~s}$ which provides only a small integration time on each spectrum (pixel). Thus the rms in the OTF spectra, $0.22 \mathrm{~K}$ in $T_{\mathrm{mb}}$ in an $88^{\prime \prime}$ beam and $3 \mathrm{~km} \mathrm{~s}^{-1}$ channel, is larger than that in the corresponding GREAT spectra.

The OTF map data presented here were processed following one of the procedures suggested by the Herschel Science Center science staff, by running the HIPE pipeline without an off-source subtraction to produce level 1 data. Then the hebCorrection routine was applied to the level 1 data to remove the HEB standing waves and afterwards we followed the procedure described in Velusamy \& Langer (2014) to produce the level 2 data and spectral line maps. The fact that hebCorrection subtracts the matching standing wave patterns from a large database of spectra eliminates the need for off-source subtraction. Thus in our analysis the processed spectral data are free from any off source contamination. We use the processed spectral line scan map data to make latitude-velocity $(b-V)$ maps of the velocity structure of the [C II] emission as a function of latitude at each of the 13 longitudes of the OTF positions. To increase the sensitivity of the $b-V$ maps we use a velocity resolution of $3 \mathrm{~km} \mathrm{~s}^{-1}$. In all the OTF $b-V$ maps the velocity feature near $-207 \mathrm{~km} \mathrm{~s}^{-1}$ is prominent, but that of the weaker $-174 \mathrm{~km} \mathrm{~s}^{-1}$ feature is less so, possibly due to the combined effect of low sensitivity and beam dilution. However, the bright $[\mathrm{C} \mathrm{II}]$ features $(>1 \mathrm{~K})$ are well traced in the OTF maps. To bring out the spatial structure of the $-207 \mathrm{~km} \mathrm{~s}^{-1}$ velocity feature we generate a [C II] integrated intensity map covering $-220 \mathrm{~km} \mathrm{~s}^{-1}$ to $-200 \mathrm{~km} \mathrm{~s}^{-1}$. This velocity range facilitates comparison with the Nobayama CO maps of Oka et al. (1998), which are presented in $10 \mathrm{~km} \mathrm{~s}^{-1}$ intervals. To generate the $[\mathrm{C} \mathrm{II}]$ map we integrate the intensities in the $b-V$ maps at each latitude pixel over the range of velocities from $-220 \mathrm{~km} \mathrm{~s}^{-1}$ to $-200 \mathrm{~km} \mathrm{~s}^{-1}$ around the [C II] spectral peak. We then use these intensities at all longitudes to generate the 2D map shown in Fig. 5. Note that while the intensities are sampled contiguously in latitude, we only have data at 13 discrete longitudes 


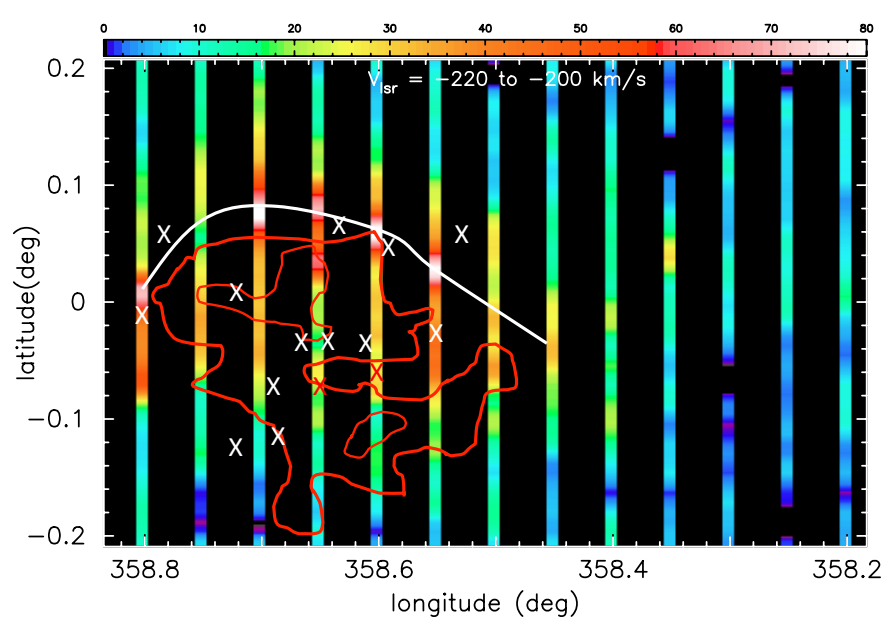

Fig. 5. Comparison of the [C II] OTF intensity maps integrated over the velocity range $-220 \mathrm{~km} \mathrm{~s}^{-1}$ to $-200 \mathrm{~km} \mathrm{~s}^{-1}$ shown as coulored strips with the CO map from Oka et al. (1998) integrated over the velocity range -220 to $-210 \mathrm{~km} \mathrm{~s}^{-1}$. The CO integrated intensity maps from Oka et al. (1998) are shown only in $10 \mathrm{~km} \mathrm{~s}^{-1}$ steps, but to improve the $\mathrm{S} / \mathrm{N}$ in the [C II] OTF maps we use a range of $20 \mathrm{~km} \mathrm{~s}^{-1}$. The CO map integrated from -210 to $-200 \mathrm{~km} \mathrm{~s}^{-1}$ shows the same extent in $b$ and the same edge at $l \sim 358.5$ as the -220 to $-210 \mathrm{~km} \mathrm{~s}^{-1}$ map, but extends further to $359^{\circ} .0$, and would give the same morphological relationship to the $[\mathrm{C} \mathrm{II}]$ contours. The color bar at the top indicates the $[\mathrm{C}$ II $]$ integrated intensity in $\mathrm{K} \mathrm{km} \mathrm{s}^{-1}$. The [C II] OTF maps sample in $b$ every 0.05 in longitude from $358^{\circ} .2$ to $358^{\circ} .8$, so generating a contour map is impractical due to the sparse sampling in $l$. Instead we have indicated by the white line the connection of the peak intensity in each strip. The $\mathrm{CO}$ contours, indicated in red, are in steps of $15 \mathrm{~K} \mathrm{~km} \mathrm{~s}^{-1}$. The [C II] is strongest slightly beyond the edge of the $\mathrm{CO}$ emission above the plane. The compact H II sources towards Sgr E (Liszt 1992) are indicated by white and red X symbols, depending on background colour.

with a pixel width of $12^{\prime \prime}$. To make the data more visible on the map we broadened their display in longitude to $60^{\prime \prime}$. There is little or no [C II] for $l<358^{\circ} .30$.

\section{Results}

The relationship between the distribution in [C II] and $\mathrm{CO}$ is brought out in Fig. 5 where we superimpose on the [C II] strip maps the $\mathrm{CO}$ integrated intensity from $-210 \mathrm{~km} \mathrm{~s}^{-1}$ to $-220 \mathrm{~km} \mathrm{~s}^{-1}$ (Oka et al. 1998). The only caveat is that the region at the edge of the $\mathrm{CMZ}$ is irregularly mapped in $\mathrm{CO}$ in the Nobayama maps. However, the AT\&T Bell Laboratories CO survey mapped the CMZ out to $l= \pm 2^{\circ}$ (see Fig. 1; Morris \& Serabyn 1996) and also shows a sharp edge in CO emission at $l \sim 358^{\circ} .5$. The $-207 \mathrm{~km} \mathrm{~s}^{-1}$ cloud is clearly seen in the positionvelocity maps from the AT\&T Bells Laboratories CO survey (see Fig. 4; Morris \& Serabyn 1996). The CO emission comes from the cloud associated with Sgr E, an active region of star formation with bright compact $\mathrm{H}$ II regions. Though our [C II] map is not contiguous in longitude (scans are spaced by $3^{\prime}$ ) the brightest emission (white in the color wedge) appears to be an arc above the plane slightly outside the lowest CO contour. The [C II] arc is most likely limb brightened emission. There does not appear to be a similar limb brightening for $b<0^{\circ}$. Although we do not have any $[\mathrm{N} \mathrm{II}]$ observations along this arc, we conclude from the presence of $[\mathrm{N} \mathrm{II}]$ along the line of sight to the $\mathrm{CO}$ cloud at $b=0^{\circ}$ that the $[\mathrm{N} \mathrm{II}]$ and $[\mathrm{C} \mathrm{II}]$ emission arise from an ionized region surrounding the cloud, but there is also a likely contribution to [C II] from a photon dominated region (PDR) sandwiched

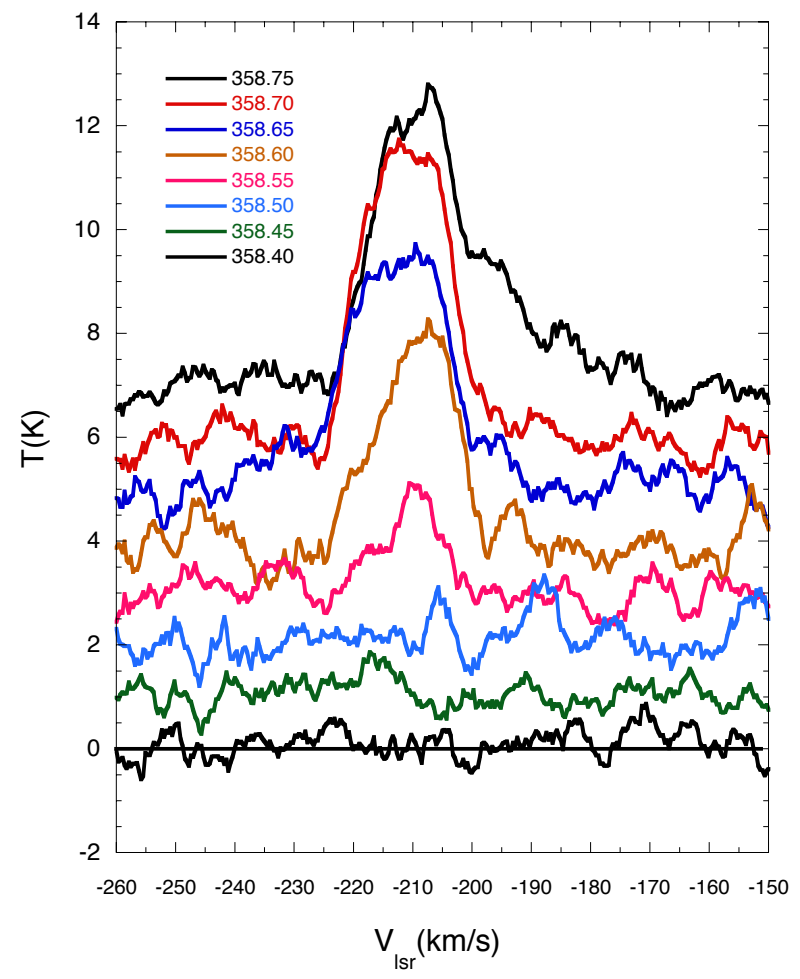

Fig. 6. $\mathrm{CO}(1-0)$ spectra at $b=0^{\circ}$ at the longitudes observed in $[\mathrm{C} \mathrm{II}]$ and $[\mathrm{N}$ II]. The data is taken from the ThrUMMS survey (uncorrected for main beam efficiency) and the inset is a key to the lines of sight observed. Moving towards the $\mathrm{CMZ}$, larger $l$, $\mathrm{CO}$ is not detected until $l \sim 358^{\circ} .55$ (although there is a hint of $\mathrm{CO}$ at $358^{\circ} .50$ ) at the inner edge of the CMZ, and that only the $-207 \mathrm{~km} \mathrm{~s}^{-1}$ component is present. The $-174 \mathrm{~km} \mathrm{~s}^{-1} \mathrm{CO}$ component is detected at $358^{\circ} .75$, the position furthest from the edge of the CMZ and it is considerably weaker there than the $-207 \mathrm{~km} \mathrm{~s}^{-1}$ component.

between the highly ionized hydrogen and dense molecular gas traced by $\mathrm{CO}$. Also indicated on the map are 15 of the $18 \mathrm{com}-$ pact H II sources detected by Liszt (1992) towards Sgr E (the remaining three are outside the mapped region). In addition to these compact $\mathrm{H}$ II regions there appears to be low level diffuse emission from $l \sim 358.8$ to $359^{\circ} 0$ between $b= \pm 0^{\circ} .1$ (see Fig. 8 in Liszt 1992). The compact H II sources have electron densities of order several hundred $\mathrm{cm}^{-3}$ and require ionizing stars of type B0 or brighter (Liszt 1992).

All six [C II] and the four [N II] spectra in Fig. 4 show emission in the velocity range -140 to $-240 \mathrm{~km} \mathrm{~s}^{-1}$. The detection of broad emission in [C II] and [N II] indicates the presence of warm, and perhaps dense, ionized gas at the edge of the CMZ. To gain further insight into the nature of the gas and its association with different interstellar medium (ISM) components we compare $[\mathrm{C}$ II $]$ and $[\mathrm{N}$ II $]$ to the $\mathrm{CO}(1-0)$ and $\mathrm{HI}$ spectra along $b=0^{\circ}$ taken from the Three-mm Ultimate Mopra Milky Way Survey ${ }^{4}$ (ThrUMMS) (Barnes et al. 2011) and the Australia Telescope Compact Array H I Galactic Center survey ${ }^{5}$ (McClure-Griffiths et al. 2012), respectively.

In Fig. 6 we plot the $\mathrm{CO}(1-0)$ spectra at $b=0^{\circ}$ along the strip observed with GREAT, $l=358^{\circ} .40$ to $358^{\circ} .75$ and $b=0^{\circ}$. The corresponding H I spectra are plotted in Fig. 7. The CO and H I spectra show that there are two different environments detected in [C II] and [N II]. The emission at $V_{\mathrm{lsr}} \sim-207 \mathrm{~km} \mathrm{~s}^{-1}$

\footnotetext{
wWw.astro.ufl.edu/thrumms

wwW.atnf.csiro.au/research/HI/sgps/GalacticCenter
} 


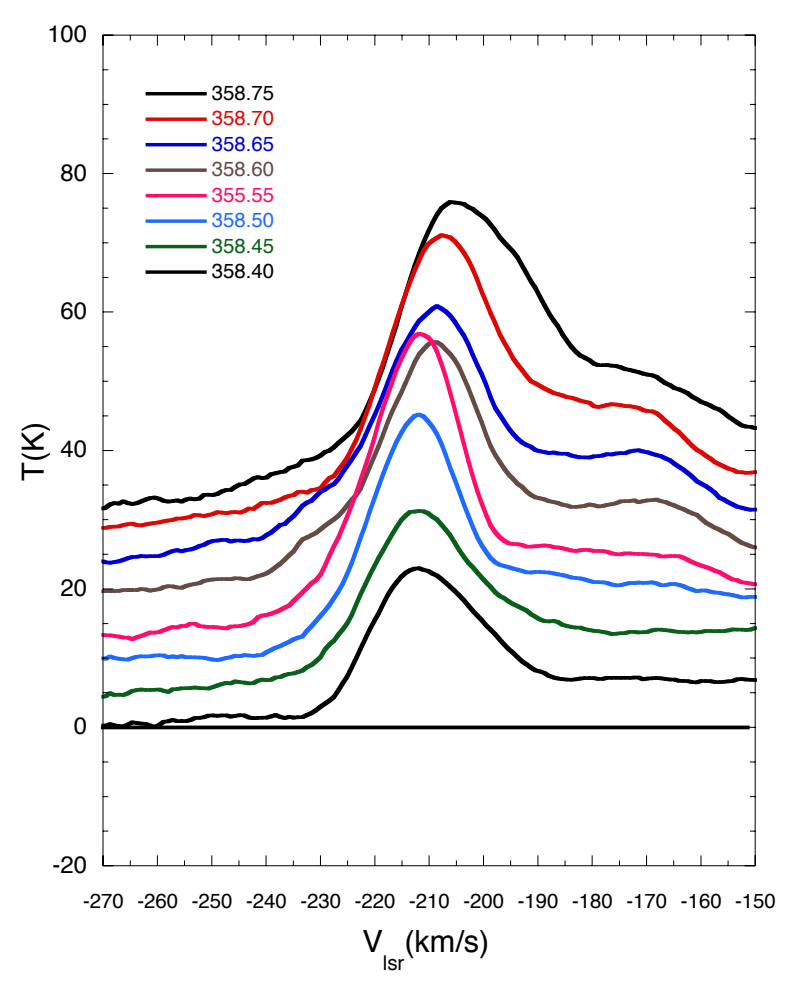

Fig. 7. H I spectra at $b=0^{\circ}$ along the latitudes observed in [C II] and [N II]. The data are taken from the ATCA H I survey (McClure-Griffiths et al. 2012) and the inset is a key to the lines of sight observed. H I is detected at all positions at $-207 \mathrm{~km} \mathrm{~s}^{-1}$ and is strongest close to Sgr E. $\mathrm{HI}$ is also detected at $-174 \mathrm{~km} \mathrm{~s}^{-1}$ but is weaker than at $-207 \mathrm{~km} \mathrm{~s}^{-1}$.

is associated with both $\mathrm{CO}$ and $\mathrm{HI}$, while that for $-174 \mathrm{~km} \mathrm{~s}^{-1}$ is associated only with $\mathrm{HI}$ until $l \sim 358^{\circ} .75$, where there is a hint of $\mathrm{CO}$ emission. The distributions of the [C II] and [N II] emission for the $-207 \mathrm{~km} \mathrm{~s}^{-1}$ component are towards the edge of a CO molecular cloud associated with Sgr E, while the $-174 \mathrm{~km} \mathrm{~s}^{-1}$ distribution is along the edge of another $\mathrm{CO}$ molecular cloud, as can be seen in the Nobayama CO maps in this velocity range (Oka et al. 1998).

To evaluate the properties of the ionized gas we derived the parameters of the [C II], [N II], and $\mathrm{CO}$ from Gaussian fits to each line component. In cases where the lines are blended we derive their line parameters using a double Gaussian fit over the velocity range $-100 \mathrm{~km} \mathrm{~s}^{-1}$ to $-300 \mathrm{~km} \mathrm{~s}^{-1}$. The intensities are given in Table 1. We calculate the corresponding $\mathrm{H}$ I intensities by integrating over the velocity ranges of the two [C II] components because we cannot get unambiguous Gaussian fits over such broad and, at low velocities, flat lines. To avoid double counting the intensities we chose $-190 \mathrm{~km} \mathrm{~s}^{-1}$ as the dividing velocity of the two components, and their intensities are given in Table 1. In general, there is a good correlation of [N II] intensity with [C II] intensity, which suggests that a significant fraction of the $[\mathrm{C}$ II $]$ emission arises in the $[\mathrm{N}$ II] region. We will use the $[\mathrm{C} \mathrm{II}]$ and $[\mathrm{N} \mathrm{II}]$ integrated intensities to characterize the properties of the ionized gas.

\subsection{Radiative transfer solutions for [C II] and [N॥]}

The relationship of the column density of $\mathrm{C}^{+}$and line intensity, $I([\mathrm{C} \mathrm{II}])$, has been extensively discussed in the literature (e.g. Goldsmith et al. 2012). The [C II] peak antenna temperatures reported here are $\ll 0.3$ times the kinetic temperature and,
Table 1. Integrated line intensities.

\begin{tabular}{lcccc}
\hline \hline LOS & $I([\mathrm{C} \mathrm{II}])^{a, b}$ & $I([\mathrm{~N} \mathrm{II}])^{c}$ & $I(\mathrm{CO})$ & $I(\mathrm{H} \mathrm{I})$ \\
\hline$V_{\mathrm{lsr}}=-207 \mathrm{~km} \mathrm{~s}^{-1}$ & & & & \\
$358.45+0.0$ & 63.4 & 26.4 & 6.7 & 1047 \\
$358.55+0.0$ & 45.4 & 15.4 & 26.6 & 1904 \\
$358.60+0.0$ & 47.6 & 7.1 & 60.6 & 2189 \\
$358.65+0.0$ & 38.6 & - & 101.9 & 2539 \\
$358.70+0.0$ & 45.4 & 12.4 & 98.9 & 2923 \\
$358.75+0.0$ & 57.4 & - & 110.5 & 3235 \\
\hline$V_{\mathrm{lsr}}=-174 \mathrm{~km} \mathrm{~s}^{-1}$ & & & & \\
$358.45+0.0$ & 21.1 & 8.2 & - & 730 \\
$358.55+0.0$ & 12.2 & 8.8 & - & 1212 \\
$358.60+0.0$ & 43.0 & 3.7 & - & 1544 \\
$358.65+0.0$ & 25.5 & - & - & 1855 \\
$358.70+0.0$ & 15.3 & 5.3 & - & 2177 \\
$358.75+0.0$ & 21.8 & - & 9.8 & 2522 \\
\hline
\end{tabular}

Notes. ${ }^{(a)}$ Integrated intensities are in units of $\mathrm{K} \mathrm{km} \mathrm{s}^{-1}$. We only report detections with a $S / N \geq 3$, see text. ${ }^{(b)}$ Typical rms noise in the [C II] integrated intensity is $\sim 1.4 \mathrm{~K} \mathrm{~km} \mathrm{~s}^{-1}$. ${ }^{(c)}$ Typical rms noise in the [N II] ntegrated intensity is $\sim 1.3 \mathrm{~K} \mathrm{~km} \mathrm{~s}^{-1}$.

as discussed by Goldsmith et al. (2012), are in the "effectively optically thin limit" in which the column density is proportional to the integrated line intensity. Therefore we have from Langer et al. (2014), Eq. (A.2), the relationship between the column density, $N\left(\mathrm{C}^{+}\right)$in $\mathrm{cm}^{-2}$ and integrated line intensity, $\int T(K) \mathrm{dv}$, in $\mathrm{K} \mathrm{km} \mathrm{s}^{-1}$,

$N\left(C^{+}\right)=2.92 \times 10^{15}\left[1+0.5 \mathrm{e}^{\Delta E / k T}\left(1+\frac{n_{\mathrm{cr}}}{n}\right)\right] I([\mathrm{C} \mathrm{III}]) \mathrm{cm}^{-2}$

where $\Delta E / k=91.25 \mathrm{~K}$ is the energy needed to excite the ${ }^{2} \mathrm{P}_{3 / 2}$ level, $n$ is the density of the collision partner of the bulk of the gas, and $n_{\mathrm{cr}}$ is the corresponding critical density. The collision rate coefficients for $\mathrm{e}, \mathrm{H}$, and $\mathrm{H}_{2}$ are summarized in Goldsmith et al. (2012) with an update for $\mathrm{H}_{2}$ in Wiesenfeld \& Goldsmith (2014). Electrons will dominate the excitation of $\mathrm{C}^{+}$where we detect $[\mathrm{NII}]$. In the ionized regions where $[\mathrm{N} \mathrm{II}]$ is detected the temperatures are high enough that we can neglect the exponential term and

$N\left(\mathrm{C}^{+}\right)=2.92 \times 10^{15}\left[1+0.5\left(1+\frac{n_{\mathrm{cr}}(\mathrm{e})}{n(\mathrm{e})}\right)\right] I([\mathrm{C} \mathrm{III}]) \mathrm{cm}^{-2}$.

The relationship of the column density of ionized nitrogen to the integrated line intensity, in units of $\mathrm{K} \mathrm{km} \mathrm{s}^{-1}$, in the optically thin limit, assuming uniform excitation along the line of sight, is given by,

$I_{u l}([\mathrm{~N} \mathrm{II}])=\int T_{u l}(K) \mathrm{dv}=\frac{h c^{3}}{8 \pi k\left(v_{u l}\right)^{2}} A_{u l} f_{u} N\left(\mathrm{~N}^{+}\right) \mathrm{cm}^{-2}$,

where $A_{u l}$ is the Einstein A-coefficient, $v_{u l}$ the transition frequency, $f_{u}$ is the fractional population of the upper state which depends on the density of the collision partner, $n, I$ is in units of $\mathrm{K} \mathrm{km} \mathrm{s}^{-1}$, and $N\left(\mathrm{~N}^{+}\right)$is the column density of ionized nitrogen. For the ${ }^{3} \mathrm{P}_{1} \rightarrow{ }^{3} \mathrm{P}_{0}$ transition at $205 \mu \mathrm{m}$ this equation reduces to

$I([\mathrm{~N} \mathrm{II}])=5.06 \times 10^{-16} f_{1} N\left(\mathrm{~N}^{+}\right)\left(\mathrm{K} \mathrm{km} \mathrm{s}^{-1}\right)$

where $f_{1}$ is the fractional population of $\mathrm{N}^{+}$in the ${ }^{3} \mathrm{P}_{1}$ state. If we assume that the [N II] emission region is uniform we can replace $N\left(\mathrm{~N}^{+}\right)$by, $n\left(\mathrm{~N}^{+}\right) L$, where $L$ is the path length in $\mathrm{cm}$,

$I([\mathrm{~N} \mathrm{II}])=5.06 \times 10^{-16} f_{1} n\left(\mathrm{~N}^{+}\right) \mathrm{L}\left(\mathrm{K} \mathrm{km} \mathrm{s}^{-1}\right)$. 
W. D. Langer et al.: Ionized gas at the edge of the central molecular zone

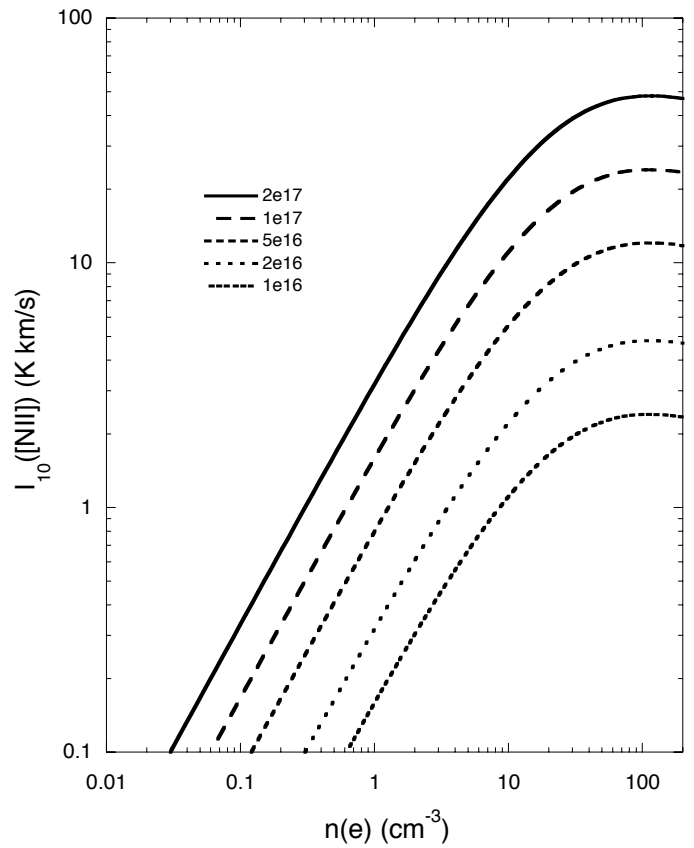

Fig. 8. Intensity of [N II] $205 \mu$ m line versus $n$ (e) for a range of ionized nitrogen column densities; the insert gives the key for the column density. The curves flatten out as the density approaches the critical density.

We can solve for $f_{1}$ in the optically thin limit from the detailed balance equations for the ${ }^{3} \mathrm{P}_{2},{ }^{3} \mathrm{P}_{1}$, and ${ }^{3} \mathrm{P}_{0}$ states along with the condition $f_{0}+f_{1}+f_{2}=1$. In ionized regions electrons dominate the excitation of $\mathrm{N}^{+}$. The collisional de-excitation rate coefficient by electrons is given by

$C_{u l}=8.63 \times 10^{-6} \frac{G(u, l)}{g_{u} T^{0.5}}$,

where $g_{u}$ is the degeneracy of the upper level and $G(u, l)$ is the temperature dependent effective collision strength (see Lennon \& Burke 1994; Hudson \& Bell 2004). We calculated the electron collisional rate coefficients using the Hudson \& Bell (2004) values for the effective collision strengths. The critical density for the 1-0 transition, where collisions out of the ${ }^{3} \mathrm{P}_{1}$ state balance the radiative transition, $\mathrm{A}_{10}$, is $n_{\mathrm{cr}}(\mathrm{e}) \sim 44 \mathrm{~cm}^{-3}$ at $8000 \mathrm{~K}$. In Fig. 8 we plot the intensity of the $205 \mu \mathrm{m}$ line as a function of the electron density using Eq. (4) for a range of ionized nitrogen column densities, $N\left(\mathrm{~N}^{+}\right)=10^{16}$ to $10^{18} \mathrm{~cm}^{-2}$. The intensity scales linearly with $n(\mathrm{e})$ up to $\sim 10-15 \mathrm{~cm}^{-3}$ beyond which it levels off as $n(\mathrm{e})$ approaches the critical density.

The $[\mathrm{N}$ II] emission comes from ionized gas and so can be used to derive the electron density directly if we know the size of the emitting region and the fractional abundance of ionized nitrogen, $x\left(\mathrm{~N}^{+}\right)=n\left(\mathrm{~N}^{+}\right) / n_{t}$, where $n_{t}=n(\mathrm{H})+2 n\left(\mathrm{H}_{2}\right)+n\left(\mathrm{H}^{+}\right)$is the total density of hydrogen nuclei including ions. To estimate $n(\mathrm{e})$ we replace the density of ionized nitrogen, $n\left(\mathrm{~N}^{+}\right)$, in Eq. (5) by $x\left(\mathrm{~N}^{+}\right) n_{t}$, and because the [N II] emission arises from highly, or perhaps completely, ionized gas we set $n_{t} \simeq n\left(\mathrm{H}^{+}\right)=n(\mathrm{e})$. For a uniform density region we can take $N(\mathrm{e})=n(\mathrm{e}) L$, where $L$ is the thickness of the emission region, and can now express Eq. (5) as

$I([\mathrm{~N} \mathrm{II}])=\int T_{10} \mathrm{~d} v=5.06 \times 10^{-16} x\left(\mathrm{~N}^{+}\right) \operatorname{Ln}(\mathrm{e}) f_{1}(n(\mathrm{e}))$,

where $I$ is in $\mathrm{K} \mathrm{km} \mathrm{s}^{-1}$. Rewriting in convenient units we obtain,

$I([\mathrm{~N} \mathrm{II}])=0.156 x_{-4}\left(\mathrm{~N}^{+}\right) L_{\mathrm{pc}} n(\mathrm{e}) f_{1}(n(\mathrm{e}))$
Table 2. Electron density and nitrogen column density.

\begin{tabular}{lcccccc}
\hline \hline$V_{\text {lsr }}{ }^{a}=$ & -207 & -207 & -207 & -174 & -174 & -174 \\
LOS & $n(\mathrm{e})^{b}$ & $N\left(\mathrm{~N}^{+}\right)^{c}$ & $N\left(\mathrm{H}^{+}\right)$ & $n(\mathrm{e})^{d}$ & $N\left(\mathrm{~N}^{+}\right)$ & $N\left(\mathrm{H}^{+}\right)$ \\
\hline $358.45^{d}$ & 20.7 & $1.5 \mathrm{e} 17$ & $9.3 \mathrm{e} 20$ & 9.9 & $7.5 \mathrm{e} 16$ & $4.6 \mathrm{e} 20$ \\
358.55 & 14.6 & $1.1 \mathrm{e} 17$ & $6.8 \mathrm{e} 20$ & 10.4 & $7.9 \mathrm{e} 16$ & $4.8 \mathrm{e} 20$ \\
358.60 & 9.1 & $6.9 \mathrm{e} 16$ & $4.2 \mathrm{e} 20$ & 6.3 & $4.8 \mathrm{e} 16$ & $2.9 \mathrm{e} 20$ \\
358.65 & - & - & - & - & - & - \\
358.70 & 12.8 & $9.7 \mathrm{e} 16$ & $5.9 \mathrm{e} 20$ & 7.7 & $5.9 \mathrm{e} 16$ & $3.6 \mathrm{e} 20$ \\
358.75 & - & - & - & - & - & - \\
\hline Average & 14.3 & $1.1 \mathrm{e} 17$ & $6.6 \mathrm{e} 20$ & 8.6 & $6.5 \mathrm{e} 16$ & $4.0 \mathrm{e} 20$ \\
\hline
\end{tabular}

Notes. ${ }^{(a)}$ In $\mathrm{km} \mathrm{s}^{-1} \cdot{ }^{(b)}$ Densities in $\mathrm{cm}^{-3} \cdot{ }^{(c)}$ Column densities in $\mathrm{cm}^{-2}$ and rounded to one decimal place. ${ }^{(d)}$ All LOS are along $b=0^{\circ}$.

where $x_{-4}$ is the fractional abundance of $\mathrm{N}^{+}$is in units of $10^{-4}$ and $L_{\mathrm{pc}}$ is in pc. Thus we can solve for the electron density, $n(\mathrm{e})$, as a function of the observed intensity of the $205 \mu \mathrm{m}$ line, given the size of the emission region and the fractional abundance of $\mathrm{N}^{+}$. At low densities, where $n(\mathrm{e})<n_{\mathrm{cr}}(\mathrm{e})$, it can be shown that

$n(\mathrm{e}) \propto\left[\frac{I(\mathrm{~N} \text { II })}{L_{\mathrm{pc}} x_{-4}\left(\mathrm{~N}^{+}\right)}\right]^{\alpha} \mathrm{cm}^{-3}$

where $0.5<\alpha<0.7$, for $n(\mathrm{e})<n_{\mathrm{cr}}(\mathrm{e})$. In the limit $n(\mathrm{e}) \ll n_{\mathrm{cr}}(\mathrm{e})$, such as found in the Warm Ionized Medium in the disk, $f_{1} \sim$ $n(\mathrm{e}) / n_{\mathrm{cr}}(\mathrm{e})$ and $\alpha=0.5$. Thus, in general, $n(\mathrm{e})$ is only moderately sensitive to uncertainties in $L$ and $x\left(\mathrm{~N}^{+}\right)$.

\subsection{The $-207 \mathrm{~km} \mathrm{~s}^{-1}$ component}

As seen in Figs. 4 and 5 there is [C II] emission along the line of sight to the CO molecular cloud with $V_{\mathrm{lsr}} \sim-207 \mathrm{~km} \mathrm{~s}^{-1}$. The peak in $[\mathrm{C} \mathrm{II}]$ intensity for this component is at $b>0^{\circ}$ at the edge of the lowest contour of $\mathrm{CO}$ and is thus consistent with limb brightened [C II] emission. Therefore the SOFIA [C II] and $[\mathrm{N}$ II $]$ spectra along $b=0^{\circ}$ likely arise from a layer of gas whose size is related to the thickness of the [C II] emission beyond the lowest $\mathrm{CO}$ contour. The thickness of the [C II] emission is about $6^{\prime}$ which corresponds to $\sim 15$ pc at the distance to the CMZ. To solve for the electron abundance in the $[\mathrm{N}$ II] regions we need, in addition to $L_{\mathrm{pc}}$ and $I([\mathrm{~N} \mathrm{II}]), x\left(\mathrm{~N}^{+}\right)$appropriate to the CMZ. To estimate the fraction of $\mathrm{N}^{+}$in the CMZ we use the nitrogen abundance Galactic gradient, $0.07 \mathrm{dex} \mathrm{kpc}^{-1}$, for $R_{\mathrm{G}}>3 \mathrm{kpc}$, derived from Rolleston et al. (2000) which leads to $x\left(\mathrm{~N}^{+}\right) \propto \exp \left(-R_{\mathrm{G}} / 6.2\right)$ (see Pineda et al. 2013, Langer et al. 2014). However because we do not have information about how it scales to smaller $R_{\mathrm{G}}$ we extrapolate this relationship to the half way point, $1.5 \mathrm{kpc}$. Using the local nitrogen abundance fraction $x\left(\mathrm{~N}^{+}\right)=5.51 \times 10^{-5}$ from Jensen et al. (2007) yields $x\left(\mathrm{~N}^{+}\right)=1.6 \times 10^{-4}$ at $R_{\mathrm{G}}=1.5 \mathrm{kpc}$. As discussed above, the solutions for $n(\mathrm{e})$ are not very sensitive to modest uncertainties in $x\left(\mathrm{~N}^{+}\right)$. The solutions for $n(\mathrm{e})$ and $N\left(\mathrm{~N}^{+}\right)$are given in Table 2 .

The electron density for the $-207 \mathrm{~km} \mathrm{~s}^{-1}$ component varies from $\sim 9$ to $21 \mathrm{~cm}^{-3}$ for the four LOS with [N II] detections and has an average value $\sim 14 \mathrm{~cm}^{-3}$. Thus the gas traced by [N II] is warm dense ionized gas, significantly denser than that derived for the typical WIM in the disk, which has $n(\mathrm{e})$ of order a few $\times 10^{-2} \mathrm{~cm}^{-3}$ (Haffner et al. 2009; Velusamy et al. 2012). In the next section we will discuss what might generate and sustain such a high density ionized gas towards the cloud associated with Sgr E. 
Table 3. $\mathrm{C}^{+}$column densities and intensities.

\begin{tabular}{lccccc}
\hline \hline LOS $^{a}$ & $N\left(\mathrm{C}^{+}\right)^{b}$ & $I_{\mathrm{H}^{+}}([\mathrm{C} \text { II }])^{c}$ & $I_{\mathrm{H}_{2}}([\mathrm{C} \mathrm{II}])$ & $N_{\mathrm{H}_{2}}\left(\mathrm{C}^{+}\right)$ & $N_{\mathrm{C}^{+}}\left(\mathrm{H}_{2}\right)^{f}$ \\
\hline$-207^{d}$ & & & & & \\
\hline 358.45 & $4.9 \mathrm{e} 17$ & 64.1 & -0.7 & - & - \\
358.55 & $3.5 \mathrm{e} 17$ & 38.5 & 7.0 & $4.2 \mathrm{e} 17$ & $8.2 \mathrm{e} 20$ \\
358.60 & $2.2 \mathrm{e} 17$ & 18.3 & 29.3 & $1.8 \mathrm{e} 18$ & $3.5 \mathrm{e} 21$ \\
358.65 & - & - & - & - & - \\
358.70 & $3.0 \mathrm{e} 17$ & 31.3 & 14.1 & $8.6 \mathrm{e} 17$ & $1.7 \mathrm{e} 21$ \\
358.75 & - & - & - & - & - \\
\hline Average & $3.4 \mathrm{e} 17$ & 38.1 & $16.8^{e}$ & $1.0 \mathrm{e} 18$ & $2.0 \mathrm{e} 21$ \\
\hline$-174^{d}$ & & & & & \\
\hline 358.45 & $2.4 \mathrm{e} 17$ & 21.1 & 0.1 & $3.3 \mathrm{e} 15$ & $6.4 \mathrm{e} 18$ \\
358.55 & $2.5 \mathrm{e} 17$ & 22.6 & -10.4 & - & - \\
358.60 & $1.5 \mathrm{e} 17$ & 9.9 & 33.1 & $2.0 \mathrm{e} 18$ & $3.9 \mathrm{e} 21$ \\
358.65 & - & - & - & - & - \\
358.70 & $1.8 \mathrm{e} 17$ & 13.4 & 1.3 & $8.2 \mathrm{e} 16$ & $1.6 \mathrm{e} 20$ \\
358.75 & - & - & - & - & - \\
\hline Average & $2.0 \mathrm{e} 17$ & 16.9 & $11.5^{e}$ & $7.0 \mathrm{e} 17$ & $1.4 \mathrm{e} 21$ \\
\hline
\end{tabular}

Notes. ${ }^{(a)}$ All LOS are at $b=0^{\circ} .{ }^{(b)}$ Column density in $\mathrm{cm}^{-2}$ and rounded to one decimal place. ${ }^{(c)}$ Intensity of $[\mathrm{C}$ II] in the [N II] emission region in $\mathrm{K} \mathrm{km} \mathrm{s}^{-1} \cdot{ }^{\left({ }^{(d)}\right.} V_{\mathrm{lsr}}$ in $\mathrm{km} \mathrm{s}^{-1}$. ${ }^{(e)}$ Negative intensities are not included. (f) Assumes $T_{k}=100 \mathrm{~K}$ and $n\left(\mathrm{H}_{2}\right)=300 \mathrm{~cm}^{-3}$.

Assuming that the nitrogen is nearly fully ionized, the column density of ionized carbon in this region is given by $N\left(\mathrm{C}^{+}\right)=$ $x\left(\mathrm{C}^{+}\right) / x\left(\mathrm{~N}^{+}\right) N\left(\mathrm{~N}^{+}\right)$. For $x\left(\mathrm{C}^{+}\right) / x\left(\mathrm{~N}^{+}\right)$we adopt the value of 3.2 (Jensen et al. 2007; Sofia et al. 2004) and the values for $N\left(\mathrm{C}^{+}\right)$ are given in Table 3 along with $N\left(\mathrm{H}^{+}\right)$. The $\mathrm{C}^{+}$column densities are of order a few $\times 10^{17} \mathrm{~cm}^{-2}$, with an average $\sim 4 \times 10^{17} \mathrm{~cm}^{-2}$.

The $[\mathrm{C}$ II $]$ intensity associated with the $[\mathrm{N}$ II $]$ region, $I_{\mathrm{H}^{+}}([\mathrm{C} \mathrm{II}])$, is calculated using the inverse of Eq. (2), from the column density $N\left(\mathrm{C}^{+}\right), n(\mathrm{e})$ derived from [N II], and assuming $T_{k}=8000 \mathrm{~K}$. The exact kinetic temperature is not critical as the column density is only weakly dependent on temperature through the collisional de-excitation rate coefficient, resulting in $n_{\mathrm{cr}}(\mathrm{e}) \sim 50 T_{4}{ }^{0.37} \mathrm{~cm}^{-3}$, where $T_{4}$ is the kinetic temperature in units of $10^{4} \mathrm{~K}$. The results are given in Table 3.

The $[\mathrm{C}$ II $]$ emission from the neutral PDR, $I_{\mathrm{H}_{2}}([\mathrm{C}$ II $])$, is the difference between the observed total $[\mathrm{C}$ II] intensity, I([C II] $)$, given in Table 1 , and the intensity calculated from the [N II] region, $I_{\mathrm{H}^{+}}([\mathrm{C} \mathrm{II}])$ (Langer et al. 2014). This quantity, $I_{\mathrm{H}_{2}}([\mathrm{C} \mathrm{II}])=$ $I([\mathrm{CII}])-I_{\mathrm{H}^{+}}([\mathrm{C} \mathrm{II}])$ arises primarily from the $\mathrm{CO}$-dark $\mathrm{H}_{2}$ gas (also called the $\mathrm{CO}-$ dark gas) and is given in Table 3. Negative or very small values of $I_{\mathrm{H}_{2}}([\mathrm{CII}])$ indicate that almost all the [C II] emission arises from the dense ionized gas. Unfortunately we do not know the kinetic temperature and hydrogen density of the CO-dark $N\left(\mathrm{H}_{2}\right)$ to calculate accurately its measured $\mathrm{C}^{+}$ column density. For illustrative purposes we calculate the column density of $\mathrm{C}^{+}$in the $\mathrm{H}_{2}$ layer, $N_{\mathrm{H}_{2}}\left(\mathrm{C}^{+}\right)$, and the corresponding column density of CO-dark $\mathrm{H}_{2}, N_{\mathrm{C}^{+}}\left(\mathrm{H}_{2}\right)=N_{\mathrm{H}_{2}}\left(\mathrm{C}^{+}\right) / x\left(\mathrm{C}^{+}\right)$ (see Langer et al. 2014), assuming $100 \mathrm{~K}$ and $300 \mathrm{~cm}^{-3}$ in Eq. (1), along with the appropriate $n_{\mathrm{cr}}\left(\mathrm{H}_{2}\right)$ from Wiesenfeld \& Goldsmith (2014). These results are listed in Table 3 for all the LOS with positive $I_{\mathrm{H}_{2}}([\mathrm{C} \mathrm{II}])$. Higher densities will result in lower column densities and vice versa.

\subsection{The $-174 \mathrm{~km} \mathrm{~s}^{-1}$ component}

The $-174 \mathrm{~km} \mathrm{~s}^{-1}[\mathrm{NII}]$ and [C II] emissions are weaker, and in some positions much weaker, than those of the $-207 \mathrm{~km} \mathrm{~s}^{-1}$ component, and are associated with $\mathrm{CO}$ only at $l=358^{\circ} .75$ in
Fig. 6. The CO maps in Oka et al. (1998) show a CO molecular cloud with an edge at this longitude and extending inwards to larger $l$. The $-174 \mathrm{~km} \mathrm{~s}^{-1}$ emission is also associated with H I emission (Fig. 7) that is weaker than that of the $-210 \mathrm{~km} \mathrm{~s}^{-1}$ cloud. We do not detect this weaker [C II] component at all the positions in the HIFI OTF maps (see discussion in Sect. 2.2) and so have no estimate of the size of the [C II] and [N II] emission region. For comparison we have calculated densities and the column densities of this component assuming the same size, $15 \mathrm{pc}$, for the [N II] emission region as that for the $-207 \mathrm{~km} \mathrm{~s}^{-1}$ cloud. These results are given in Tables 2 and 3. The solutions for the $-174 \mathrm{~km} \mathrm{~s}^{-1}$ component yield slightly lower average electron densities and column densities than for the $-207 \mathrm{~km} \mathrm{~s}^{-1}$ component.

\section{Discussion}

The analysis of $[\mathrm{N} \mathrm{II}]$ emission at the edge of the CMZ reveals a hot dense plasma with an electron abundance ranging from $\sim 6$ to $21 \mathrm{~cm}^{-3}$. The densities we derive are much larger than those characteristic of the disk's WIM, but are consistent with those derived for very bright nebula with numerous luminous O-type stars. For example, Oberst et al. (2011) studied the Carina nebula, which has a very large UV flux, using emission from the [N II] $205 \mu \mathrm{m}$ and $122 \mu \mathrm{m}$ lines along with an excitation model ${ }^{6}$, and find values of $n(\mathrm{e})$ ranging from a few to over $100 \mathrm{~cm}^{-3}$. In the discussion below we will use the results from our analysis of [N II] and [C II] emission to characterize some of the possible energy sources that can maintain such a hot dense ionized plasma.

\subsection{Ionization of nitrogen}

Nitrogen has an ionization potential of $14.534 \mathrm{eV}$, higher than that of hydrogen at $13.598 \mathrm{eV}$, and in the ISM can be ionized by cosmic rays, X-rays, extreme ultraviolet (EUV) photons with wavelengths shortward of $853.06 \AA$, electron collisional ionization, or via charge exchange with protons $\left(\mathrm{H}^{+}\right)$at energies $\geq 0.936 \mathrm{eV}$, the energy difference between the ionization of $\mathrm{H}$ and $\mathrm{N}$. In a nearly fully ionized region $\mathrm{N}^{+}$is destroyed primarily via electron radiative and dielectronic recombination, and in partially ionized regions also via exothermic charge exchange with $\mathrm{H}$ atoms. We can estimate the required ionization rate to sustain a highly ionized $\mathrm{N}^{+}$gas by calculating the electron recombination rate for the solutions for $n(\mathrm{e})$ given in Table 2. The recombination rate coefficients are taken from the UMIST data base (McElroy et al. 2013) and Badnell (2006) for radiative recombination and Badnell et al. (2003) for dielectronic recombination. (In molecular hydrogen clouds $\mathrm{N}^{+}$reacts rapidly with $\mathrm{H}_{2}$ and initiates a chain of reactions producing nitrogen bearing molecules, but this channel is not relevant in the WIM, WNM, or $\mathrm{H}$ II regions.) Charge exchange with $\mathrm{H}$ has a reaction rate coefficient at $8000 \mathrm{~K} \sim 5.5 \times 10^{-14} \mathrm{~cm}^{3} \mathrm{~s}^{-1}$ (see Table 2 of Lin et al. 2005 ) and when compared with recombination with electrons $\sim 8 \times 10^{-13} \mathrm{~cm}^{3} \mathrm{~s}^{-1}$, we see that electron recombination dominates for $n(\mathrm{e}) / n(\mathrm{H})>0.2$. To get an estimate of the ionization rate needed to sustain a highly ionized gas we neglect the charge exchange of $\mathrm{N}^{+}$with $\mathrm{H}$ in the regime where the gas is highly ionized and consider only recombination by electrons. For large electron densities in the range 5 to $25 \mathrm{~cm}^{-3}$ such as derived here, the recombination rate ranges from $4 \times 10^{-12}$ to $2 \times 10^{-11} \mathrm{~s}^{-1}$.

\footnotetext{
6 With two transitions the electron density can be derived without having to assume a characteristic size for the emission region.
} 
Sustaining a high ionization fraction $(\geq 0.5)$ requires an ionization rate comparable to or greater than these values. For typical WIM conditions in the disk, $n(\mathrm{e}) \sim 10^{-2} \mathrm{~cm}^{-3}$, the ionization rate required to keep nitrogen highly ionized is two to three orders of magnitude lower. In the following we discuss the likely sources of ionization at the edge of the CMZ. We exclude electron collisional ionization because, due to the high ionization potential of $\mathrm{N}$, it requires a very hot electron gas with kinetic temperatures of order $10^{5} \mathrm{~K}$, a condition met only in the HIM portion of the CMZ.

\subsubsection{Cosmic ray ionization}

Goto et al. (2014) have detected $\mathrm{H}_{3}{ }^{+}$in the $\mathrm{CMZ}$ and use its abundance to derive a lower limit on the hydrogen ionization rate, $\zeta_{\mathrm{H}}>1 \times 10^{-15} \mathrm{~s}^{-1}$, which they suggest could come from cosmic rays or X-rays. If the ionization is due to cosmic rays, it is about ten to thirty times larger than the cosmic ray ionization rate in the disk (Indriolo \& McCall 2012). The cosmic ray ionization rate of nitrogen (including the secondary photons generated by cosmic rays interacting with hydrogen) is about a factor of two larger than that of hydrogen (McElroy et al. 2013), few $\times 10^{-15} \mathrm{~s}^{-1}$, but is much lower than the values needed to offset the recombination by electrons. Although regions of intense cosmic ray ionization may exist with ionization rates $\sim 10^{-14} \mathrm{~s}^{-1}$ (Bayet et al. 2011; Meijerink et al. 2011), rates $\geq 10^{-12} \mathrm{~s}^{-1}$ would be required for cosmic rays to explain high fractional nitrogen ionization at densities $n(\mathrm{e}) \geq 1 \mathrm{~cm}^{-3}$. Indeed Meijerink et al. (2011) consider rates almost that large in their models of photon dominated regions (PDRs) but only consider high atomic hydrogen densities, $n(\mathrm{H}) \geq 10^{3} \mathrm{~cm}^{-3}$ such that the fractional ionization is small. Their models cannot be applied to the ionized gas probed by our [N II] observations ${ }^{7}$.

\subsubsection{X-ray ionization}

The CMZ has several different sources of X-rays, including an accreting black hole at the center, a bright X-ray source 1E1740.7-2942 located $l \sim 359^{\circ} .1$ and $b \sim-0.1$ (e.g. Heindl et al. 1993, Wang et al. 2002), extended diffuse X-ray emission (Koyama et al. 2007), as well as over 9000 X-ray point sources (Muno et al. 2009) some of which lie at the edge of the CMZ between $l=358^{\circ} .9$ and $359^{\circ} .0$ around $b \sim 0^{\circ}$ and could provide the $>10^{-15} \mathrm{~s}^{-1}$ ionization rate of hydrogen required to explain the $\mathrm{H}_{3}{ }^{+}$observations of Goto et al. (2014). (Unfortunately, these Chandra observations of the CMZ do not extend into the Sgr E region.) However, unlike ionization by cosmic rays, the cross section for ionization of heavy atoms by X-rays can be much larger than hydrogen, primarily due to $\mathrm{K}$ shell ionization at X-ray energies $>0.4 \mathrm{keV}$, and thus X-rays could play an important role in ionizing nitrogen. For example, at $1 \mathrm{keV}$ the ionization cross section of nitrogen is $\sim 7 \times 10^{-20} \mathrm{~cm}^{-2}$ (Verner et al. 1993) over three orders of magnitude larger than that for hydrogen (Goto et al. 2014; Wilms et al. 2000). Therefore, if the X-ray ionization rate needed to explain the production of $\mathrm{H}_{3}{ }^{+}$ is $\sim$ few $\times 10^{-15} \mathrm{~s}^{-1}$, then the corresponding ionization rate for nitrogen will be of order a few $\times 10^{-12} \mathrm{~s}^{-1}$, sufficient to explain the observed large fractional ionization of nitrogen at $n(\mathrm{e}) \sim 5$

\footnotetext{
7 Note that Meijerink et al. (2011) calculate the $\mathrm{N}^{+}$fractional abundance, $x\left(\mathrm{~N}^{+}\right)=n\left(\mathrm{~N}^{+}\right) / n\left(\mathrm{H}+2 \mathrm{H}_{2}\right)$, as $\leq 10^{-7}$, which is a low ionization scenario. They also calculate the line intensities of the [N II] $205 \mu \mathrm{m}$ and $122 \mu \mathrm{m}$ lines, but their predictions fall far below the measured intensities given in Table 1.
}

to $25 \mathrm{~cm}^{-3}$. The penetration depth of $1 \mathrm{keV} \mathrm{X}$-rays is a column density $N\left(\mathrm{H}+2 \mathrm{H}_{2}\right) \sim 10^{22} \mathrm{~cm}^{-2}$, which is sufficient to penetrate the diffuse gas and envelopes of clouds in the CMZ (Goto et al. 2014). Whether X-rays are the primary ionization source or not depends on the details of the distribution and luminosity of the $\mathrm{X}$-ray sources, and we do not have sufficient information to make this determination.

\subsubsection{Photoionization}

The photoionization cross section $\sigma$ for ionizing nitrogen ranges from about $10^{-17}$ to $1.5 \times 10^{-17} \mathrm{~cm}^{2}$ from the threshold at $\sim 853 \AA$ to $500 \AA$ (Samson \& Angel 1990). The total photoionization rate is given by $\int_{\lambda<853 \AA} \sigma(\lambda) \Phi(\lambda) \mathrm{d} \lambda$, where $\Phi(=4 \pi J)$ is the incident flux integrated in units of photons $\mathrm{cm}^{-2} \mathrm{~s}^{-1}$. It has been estimated that to maintain the ionization of hydrogen in the WIM one needs an ionizing flux of Lyman continuum photons $>10^{5}$ photons $\mathrm{cm}^{-2} \mathrm{~s}^{-1}$ (Haffner et al. 2009). Reynolds et al. (1995) estimate an incident Lyman continuum flux $4 \pi J \sim 2 \times 10^{6}$ to $9 \times 10^{6}$ photons $\mathrm{cm}^{-2} \mathrm{~s}^{-1}$, sufficient to explain the ionization in the WIM. However nitrogen is ionized by EUV photons beyond the Lyman limit, for which the flux must be determined from models incorporating a distribution of $\mathrm{O}$ and $\mathrm{B}$ stars and attenuation by the surrounding ISM, including considerations of the clumpiness of the ISM (cf. Haffner et al. 2009). In general the flux drops sharply beyond the Lyman limit, as can be seen in the model of a spectrum of a star cluster in Fig. 17 of Kaufman et al. (2006), where the spectral flux (in ergs/s/ $\mathrm{Hz} / \mathrm{sr} / \mathrm{cm}^{2}$ ) drops by a factor of 10 at the Lyman edge and by another factor of 10 by $\sim 400 \AA$. We can estimate the flux needed to sustain a high nitrogen ionization fraction by balancing photoionization with electron recombination,

$n\left(\mathrm{~N}^{+}\right) / n\left(\mathrm{~N}^{0}\right)=\frac{\int_{\lambda<853 \AA} \phi(\lambda) \sigma(\lambda) \mathrm{d} \lambda}{k_{r}(\mathrm{e}) n(\mathrm{e})}$

where $k_{r}(\mathrm{e})$ is the electron recombination rate coefficient, $n\left(\mathrm{~N}^{0}\right)$ is the neutral nitrogen number density, and $\phi$ the photon flux as a function of wavelength. This equation can be simplified by assuming that the ionization cross section is roughly constant over the energy range of interest (see above), so $n\left(\mathrm{~N}^{+}\right) / n\left(\mathrm{~N}^{0}\right)$ $\sim(\Phi \sigma) /\left(k_{r} n(\mathrm{e})\right)$, where $\Phi$ is the total photon flux in $\mathrm{cm}^{2} \mathrm{~s}^{-1}$. Therefore the fraction of ionized nitrogen is

$f\left(\mathrm{~N}^{+}\right)=\frac{\Phi \sigma}{k_{r} n(e)+\Phi \sigma}$.

For example, to sustain $f\left(\mathrm{~N}^{+}\right)=0.5$ in dense ionized gas with $T=8000 \mathrm{~K}$ at $n(e)=1,10$, and $20 \mathrm{~cm}^{-3}$ requires an EUV flux $\sim 6.5 \times 10^{4}, 6.5 \times 10^{5}$, and $1.3 \times 10^{6}$ photons $\mathrm{cm}^{-2} \mathrm{~s}^{-1}$, respectively. For a nearly fully ionized nitrogen regime the required intensity of the EUV field increases sharply. For example, to maintain $f\left(\mathrm{~N}^{+}\right)=0.9$ requires an order of magnitude greater flux than for $f\left(\mathrm{~N}^{+}\right)=0.5$, which can be found near young massive stellar clusters (Kaufman et al. 2006). Rodríguez-Fernández et al. (2004) estimate that the PDRs in the CMZ are illuminated by a far-UV radiation field about a factor of $10^{3}$ larger than the local ISM, and if this increase extends to the EUV it would be sufficient to explain the ionization of nitrogen. One constraint on the flux distribution near the $-207 \mathrm{~km} \mathrm{~s}^{-1}$ Sgr E feature comes from observations of carbon and helium recombination lines. Wenger et al. (2013) detected carbon recombination lines in two of the eight Sgr E H II sources detected in hydrogen recombination lines, but did not detect any helium recombination 
lines. This result indicates that the flux at wavelengths short of $504.26 \AA$ (energies $\geq 24.59 \mathrm{eV}$ ) is small.

\subsubsection{Proton charge exchange ionization}

The last important ionization mechanism is charge exchange with energetic protons. In a highly ionized hydrogen gas, $n\left(\mathrm{H}^{+}\right)>50 n(\mathrm{H})$, we can neglect the neutralization of $\mathrm{N}^{+}$by charge exchange with $\mathrm{H}$ atoms, which then leaves only recombination with electrons to destroy $\mathrm{N}^{+}$, yielding the ratio of ionized to neutral nitrogen,

$f\left(\mathrm{~N}^{+}\right)=n\left(\mathrm{~N}^{+}\right) / n\left(\mathrm{~N}^{0}\right)=\frac{k_{x}(T) n\left(H^{+}\right)}{k_{r} n(e)}$,

where the charge exchange reaction rate coefficient, $k_{x}(T)$ is a Maxwellian average of the cross section over the electron velocity distribution (Lin et al. 2005). The value of $k_{x}(T)$ is an exponential function of temperature because only protons with energies $\gtrsim 0.94 \mathrm{eV}$ can undergo charge exchange. To a very good approximation, $n(\mathrm{e}) \simeq n\left(\mathrm{H}^{+}\right)$and the fractional abundance of nitrogen is

$f\left(\mathrm{~N}^{+}\right)=\frac{k_{x}(T)}{k_{x}(T)+k_{r}(T)}$,

which is independent of $n(\mathrm{e})$ and $n\left(\mathrm{H}^{+}\right)$. However, the fraction $f\left(\mathrm{~N}^{+}\right)$is very sensitive to the temperature of the plasma because of the endothermic nature of the reaction. The charge exchange process for $\mathrm{N}^{+}+\mathrm{H} \rightarrow \mathrm{N}+\mathrm{H}^{+}$has been measured (Stebbings et al. 1960), but only above $\sim 30 \mathrm{eV}$, and the low energy cross sections must be calculated theoretically. Lin et al. (2005) have made the most recent calculations of the charge transfer reaction with ground state nitrogen, $\mathrm{H}^{+}+\mathrm{N} \leftrightarrow \mathrm{H}+\mathrm{N}^{+}$, and use these to calculate the rate coefficients, $k_{x}$ for forward and reverse reactions. The results for the charge exchange reaction rate coefficients are given in their Table 2 and can be used to calculate the fractional nitrogen ionization as a function of kinetic temperature.

However, one must be cautious here, because the theoretical rate coefficients derived by Lin et al. (2005) for $\mathrm{H}^{+}+\mathrm{N} \rightarrow \mathrm{N}^{+}$ $+\mathrm{H}$ are lower than those of Steigman et al. (1971), Butler \& Dalgarno (1979), Kingdon \& Ferland (1996) (see Fig. 7 in Lin et al. 2005) below $2 \times 10^{4} \mathrm{~K}$, which is the relevant temperature regime for the WIM and H II regions. We solved Eq. (13) for the rates derived by Lin et al. (2005) and Kingdon \& Ferland (1996) as a function of temperature and these are plotted in Fig. 9. Using the reaction rates from Kingdon \& Ferland (1996) leads to a high fractional abundance at temperatures consistent with those measured in the WIM and H II regions, for temperatures 4000 to $10000 \mathrm{~K}$. However the rate coefficients from Lin et al. (2005) lead to a much lower ionization fraction of nitrogen at these temperatures, and require temperatures $\gtrsim 15000 \mathrm{~K}$ to ionize nitrogen efficiently via $\mathrm{H}^{+}$charge exchange. Thus, depending on which calculation of $k_{x}$ is correct will determine whether or not charge exchange with protons can sustain a high ionization fraction of $\mathrm{N}^{+}$in the $[\mathrm{N} \mathrm{II}]$ region.

\subsection{Conclusions}

From our analysis of [N II] emission at the edge of the CMZ near Sgr E we find evidence for dense, hot, highly ionized gas. The association with $[\mathrm{C} \mathrm{II}]$ and $\mathrm{CO}$ indicates that this dense ionized gas surrounds the PDRs associated with two molecular clouds, one

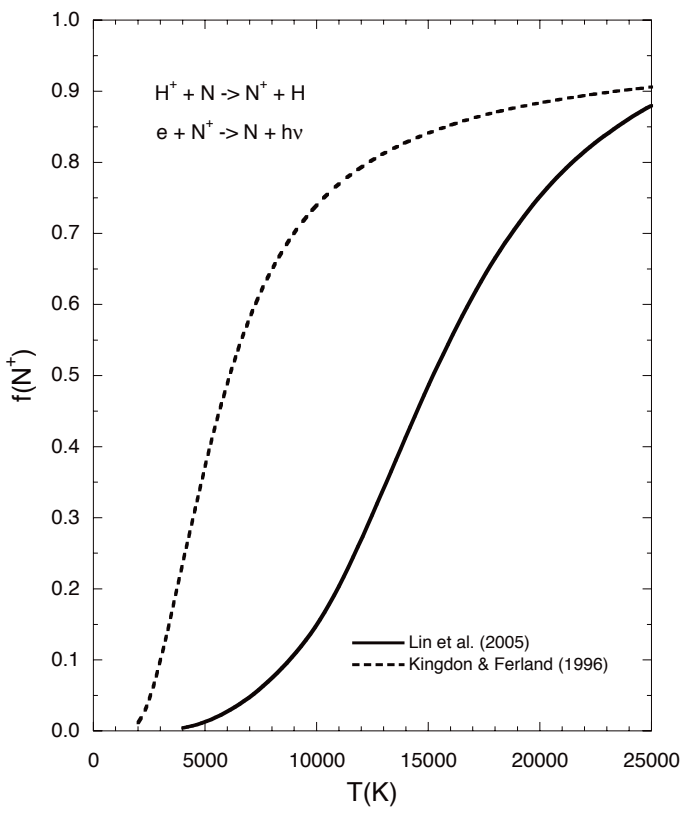

Fig. 9. Fractional nitrogen abundance as a function of kinetic temperature due to production by proton charge exchange and destruction by electron recombination. Two charge exchange reaction rate coefficients are plotted; the solid line is from Lin et al. (2005) and the dashed line from Kingdon \& Ferland (1996).

with $V_{\mathrm{lsr}} \sim-207 \mathrm{~km} \mathrm{~s}^{-1}$ and the other $\sim-174 \mathrm{~km} \mathrm{~s}^{-1}$. The electron densities, $n(\mathrm{e}) \sim 5$ to $21 \mathrm{~cm}^{-3}$, are considerably higher than those in the WIM of the disk, but consistent with those expected for bright diffuse $\mathrm{H}$ II regions, such as the Carina nebula (Oberst et al. 2011). The electron densities are considerably lower than those suggested by Lazio \& Cordes (1998), $n(\mathrm{e}) \gtrsim 10^{3} \mathrm{~cm}^{-3}$, if the photoionized layers of clouds are responsible for the scattering of radio waves in the CMZ. However, they are consistent with their other suggestion that the scattering could be due to the interface with a hot ionized medium with $n(\mathrm{e}) \sim 5-50 \mathrm{~cm}^{-3}$ and $T_{k}(\mathrm{e}) \sim 10^{5}-10^{6} \mathrm{~K}$. We have no information about the kinetic temperature from our observations, and have assumed typical WIM kinetic temperatures $\sim 8000 \mathrm{~K}$. Furthermore, our results apply to the ionized regions around the molecular cloud and if these are indeed the source of the scattering of radio waves, then they occupy a small filling factor in the CMZ and may not be representative of the electron density in the bulk of the volume.

There are three ways to sustain such a dense highly ionized hydrogen gas with large nitrogen ionization fraction. One is to have a strong EUV radiation field with a flux of order $10^{6}$ to $10^{7}$ photons $\mathrm{cm}^{-2} \mathrm{~s}^{-1}$, another is to have a high enough kinetic temperature for rapid proton charge exchange with nitrogen, and the third is if there is a flux of X-rays sufficient to ionize nitrogen in the diffuse gas. In the case of photoionization and X-ray ionization it is not necessary for the gas to be very hot. For charge exchange, a gas temperature of order $5000 \mathrm{~K}$ suffices for the rates from Kingdon \& Ferland (1996), but a much higher temperature of order $15000 \mathrm{~K}$ is required if the rates calculated by Lin et al. (2005) are correct. We are not able to distinguish which one of these processes dominates the ionization of the gas observed in [N II], or whether all three contribute. The asymmetry in the [C II] limb brightening in $b>0^{\circ}$ versus $b<0^{\circ}$ may be indicative of an asymmetry in the distribution of ionizing sources. However we lack a complete survey of the Sgr E region in X-rays or EUV from which to draw any firm conclusions. 
The edge of the CMZ extends a little farther outwards (smaller $l$ ) in ionized components than in CO. The ThrUUMS CO map, Fig. 6, detects CO at $-207 \mathrm{~km} \mathrm{~s}^{-1}$ out to $l=358^{\circ} .50$, whereas the GREAT [C II] and [N II] observations show emission out to at least $358^{\circ} .45$, a difference of $7 \mathrm{pc}$. Whether the CMZ extends any further cannot be determined as the GREAT observations did not extend to smaller values of $l$, and the HIFI OTF maps, which extend to $358^{\circ} .20$, are less sensitive than the GREAT [C II] data and show only a hint of [C II] at $-207 \mathrm{~km} \mathrm{~s}^{-1}$ extending to $358^{\circ} .35$, a distance of $14 \mathrm{pc}$ from the $\mathrm{CO}$ edge.

We have also estimated the column density of CO-dark $\mathrm{H}_{2}$ from $[\mathrm{C} \mathrm{II}]$ observations and find an average column density of order $1-2 \times 10^{21} \mathrm{~cm}^{-2}$. Models of massive molecular clouds (Wolfire et al. 2010; Bolatto et al. 2013) predict such column densities, somewhat independent of the mass of the cloud and of the intensity of the UV field. Observations of [C II] and CO towards a large ensemble of molecular clouds in the Galactic disk (Langer et al. 2014) are consistent with this model. Thus clouds in the $\mathrm{CMZ}$ and disk have similar $\mathrm{CO}$-dark $\mathrm{H}_{2}$ layers despite differences in UV radiation field and density.

\section{Summary}

We have explored the ionized gas at the edge of the CMZ near Sgr E, combining deep integrations of spectrally resolved [C II] $(158 \mu \mathrm{m})$ and [N II] $(205 \mu \mathrm{m})$ at six lines of sight along $b=0^{\circ}$ made with the GREAT instrument on SOFIA and HIFI spectrally resolved [C II] on-the-fly strip maps in $b$ at thirteen longitudes spaced 0.05 apart. We detect two distinct features in the [C II] and [N II] data, one on the line of sight to a $\mathrm{CO}$ molecular cloud at $V_{\mathrm{lsr}} \sim-207 \mathrm{~km} \mathrm{~s}^{-1}$, associated with $\mathrm{Sgr} \mathrm{E}$, and the other at $\sim-174 \mathrm{~km} \mathrm{~s}^{-1}$ outside the edge of another CO cloud.

We find that the brightest emission in the HIFI strip maps toward the cloud at $-207 \mathrm{~km} \mathrm{~s}^{-1}$ forms an arc of [C II] emission above the plane at the edge of the lowest CO contours. It likely represents limb brightened emission in a highly ionized layer outside the molecular cloud. We also detected [N II] emission at a few positions in this edge, and both [C II] and [N II] have very broad lines there with $\Delta V$ of order 25 to $35 \mathrm{~km} \mathrm{~s}^{-1}$. The electron density in this region was derived from [N II] emission and a radiative transfer model, assuming a characteristic size. We find $n(\mathrm{e}) \sim 9$ to $21 \mathrm{~cm}^{-3}$, about three orders of magnitude higher than that characterizing the disk's warm ionized medium (WIM), much smaller than those of compact H II regions, but consistent with densities derived from excitation analysis of [N II] in bright extended H II regions, such as the Carina nebula (Oberst et al. 2011). The electron densities determined from the [N II] for the ionized envelope of the cloud associated with Sgr E are lower than those suggested to explain the scattering of radio waves used to derive the average electron density distribution in the $\mathrm{CMZ}$ if the scattering comes from the ionized envelope (Lazio \& Cordes 1998).

The ionization of such a dense region requires a large flux of EUV radiation, and/or X-ray flux, and/or high enough temperatures to allow rapid charge exchange with protons via the endothermic reaction, $\mathrm{H}^{+}+\mathrm{N} \rightarrow \mathrm{H}+\mathrm{N}^{+}$. The presence of abundant $\mathrm{N}^{+}$requires this region to be hot, and the heating could be photoelectron emission from the far-UV, EUV, and X-ray radiation fields, dissipation of strong magnetic turbulence, or shock heating of supersonic turbulence. This region has many compact bright $\mathrm{H}$ II sources, indicating that it is an active massive star formation region, and such stars have the capacity to ionize and heat the gas in this region. The CMZ is also a source of diffuse X-rays and discrete X-ray sources that could provide the X-ray flux to ionize the nitrogen (as well as the hydrogen), but it depends on details of the distribution and luminosity of the X-rays. Finally, we estimate that this $\mathrm{CO}$ cloud has a layer of $\mathrm{CO}-$ dark $\mathrm{H}_{2}$ with column density $\sim 1-2 \times 10^{21} \mathrm{~cm}^{-2}$, consistent with theoretical models and observations of clouds in the disk.

We also detect ionized gas at a velocity $\sim-174 \mathrm{~km} \mathrm{~s}^{-1}$ and the lines of sight observed in [C II] and [N II] with GREAT have $\mathrm{H}$ I but no CO except except at $l=358^{\circ} .75$ which turns out to be the edge of a molecular cloud mapped in CO (Oka et al. 1998). However this [C II] emission is weak and the number of [C II] detections in the HIFI map is insufficient to determine the size of the ionized envelope for this cloud. Therefore to estimate the properties of the envelope we assumed the same thickness as that for the $-207 \mathrm{~km} \mathrm{~s}^{-1}$ cloud. An analysis of the [N II] emission using parameters similar to those for the $-207 \mathrm{~km} \mathrm{~s}^{-1}$ cloud suggests that it too is a hot high density ionized gas with $n(\mathrm{e}) \sim 6$ to $10 \mathrm{~cm}^{-3}$. The physical conditions in this ionized component are likely to be similar to those at the edge of the molecular cloud at $\sim-207 \mathrm{~km} \mathrm{~s}^{-1}$ and have similar heating and ionizing sources. Larger scale maps of spectrally resolved [C II] and [N II] will be needed to understand further the role of the ionized gas at the edge of the CMZ. Finally, the CMZ is seen to extend further out in ionized gas from the $\mathrm{CO}$ edge by upwards of at least 7 to $14 \mathrm{pc}$.

Acknowledgements. This work is based in part on observations made with the NASA/DLR Stratospheric Observatory for Infrared Astronomy (SOFIA). SOFIA is jointly operated by the Universities Space Research Association, Inc., under NASA contract NAS2-97001, and the Deutsches SOFIA Institut (DSI) under DLR contract 50 OK 0901 to the University of Stuttgart. We would like to thank Drs. R. Güsten and G. Sandell for their support of the observations and for keeping in close contact with us during the flights to adjust the observing plan as needed. In addition we owe a special thanks to Dr. David Teyssier for clarifications regarding the hebCorrection tool. We also thank an anonymous referee for comments and suggestions that improved the discussion in our paper. This work was performed at the Jet Propulsion Laboratory, California Institute of Technology, under contract with the National Aeronautics and Space Administration.

\section{References}

Anderson, L. D., Bania, T. M., Balser, D. S., \& Rood, R. T. 2011, ApJS, 194, 32

Anderson, L. D., Bania, T. M., Balser, D. S., \& Rood, R. T. 2012, ApJ, 754, 62 Ao, Y., Henkel, C., Menten, K. M., et al. 2013, A\&A, 550, A135

Badnell, N. R. 2006, A\&A, 447, 389

Badnell, N. R., O'Mullane, M. G., Summers, H. P., et al. 2003, A\&A, 406, 1151 Barnes, P. J., Yonekura, Y., Fukui, Y., et al. 2011, ApJS, 196, 12

Bayet, E., Williams, D. A., Hartquist, T. W., \& Viti, S. 2011, MNRAS, 414, 1583 Bolatto, A. D., Wolfire, M., \& Leroy, A. K. 2013, ARA\&A, 51, 207

Butler, S. E., \& Dalgarno, A. 1979, ApJ, 234, 765

Cordes, J. M., \& Lazio, T. J. W. 2002, unpublished [arXiv: astro-ph/0207156]

Cordes, J. M., \& Lazio, T. J. W. 2003, unpublished [arXiv: astro-ph/0301598]

Dame, T. M., Hartmann, D., \& Thaddeus, P. 2001, ApJ, 547, 792

de Graauw, T., Helmich, F. P., Phillips, T. G., et al. 2010, A\&A, 518, L6

Ferrière, K., Gillard, W., \& Jean, P. 2007, A\&A, 467, 611

Fujishita, M., Torii, K., Kudo, N., et al. 2009, PASJ, 61, 1039

Fukui, Y., Yamamoto, H., Fujishita, M., et al. 2006, Science, 314, 106

Goldsmith, P. F., Langer, W. D., Pineda, J. L., \& Velusamy, T. 2012, ApJS, 203, 13

Goto, M., Geballe, T. R., Indriolo, N., et al. 2014, ApJ, 786, 96

Gray, A. D. 1994, MNRAS, 270, 822

Guan, X., Stutzki, J., Graf, U. U., et al. 2012, A\&A, 542, L4

Haffner, L. M., Dettmar, R.-J., Beckman, J. E., et al. 2009, Rev. Mod. Phys., 81, 969

Heindl, W. A., Cook, W. R., Grunsfeld, J. M., et al. 1993, ApJ, 408, 507

Heyminck, S., Graf, U. U., Güsten, R., et al. 2012, A\&A, 542, L1

Hudson, C. E., \& Bell, K. L. 2004, MNRAS, 348, 1275

Indriolo, N., \& McCall, B. J. 2012, ApJ, 745, 91 
Jackson, J. M., Heyer, M. H., Paglione, T. A. D., \& Bolatto, A. D. 1996, ApJ, 456, L91

Jensen, A. G., Rachford, B. L., \& Snow, T. P. 2007, ApJ, 654, 955

Kaufman, M. J., Wolfire, M. G., \& Hollenbach, D. J. 2006, ApJ, 644, 283

Kingdon, J. B., \& Ferland, G. J. 1996, ApJS, 106, 205

Koyama, K., Hyodo, Y., Inui, T., et al. 2007, PASJ, 59, 245

Langer, W. D., Velusamy, T., Pineda, J. L., et al. 2010, A\&A, 521, L17

Langer, W. D., Velusamy, T., Pineda, J. L., Willacy, K., \& Goldsmith, P. F. 2014, A\&A, 561, A122

Lazio, T. J. W., \& Cordes, J. M. 1998, ApJ, 505, 715

Lennon, D. J., \& Burke, V. M. 1994, A\&AS, 103, 273

Lin, C. Y., Stancil, P. C., Gu, J. P., Buenker, R. J., \& Kimura, M. 2005, Phys. Rev. A, 71, 062708

Liszt, H. S. 1992, ApJS, 82, 495

Martin, C. L., Walsh, W. M., Xiao, K., et al. 2004, ApJS, 150, 239

McClure-Griffiths, N. M., Dickey, J. M., Gaensler, B. M., et al. 2012, ApJS, 199, 12

McElroy, D., Walsh, C., Markwick, A. J., et al. 2013, A\&A, 550, A36

Meijerink, R., Spaans, M., Loenen, A. F., \& van der Werf, P. P. 2011, A\&A, 525, A119

Mills, E. A. C., \& Morris, M. R. 2013, ApJ, 772, 105

Morris, M., \& Serabyn, E. 1996, ARA\&A, 34, 645

Muno, M. P., Bauer, F. E., Baganoff, F. K., et al. 2009, ApJS, 181, 110

Nakagawa, T., Yui, Y. Y., Doi, Y., et al. 1998, ApJS, 115, 259

Oberst, T. E., Parshley, S. C., Nikola, T., et al. 2011, ApJ, 739, 100

Oka, T., Hasegawa, T., Sato, F., Tsuboi, M., \& Miyazaki, A. 1998, ApJS, 118, 455
Pilbratt, G. L., Riedinger, J. R., Passvogel, T., et al. 2010, A\&A, 518, L1

Pineda, J. L., Langer, W. D., Velusamy, T., \& Goldsmith, P. F. 2013, A\&A, 554, A103

Reynolds, R. J., Tufte, S. L., Kung, D. T., McCullough, P. R., \& Heiles, C. 1995, ApJ, 448, 715

Rodríguez-Fernández, N. J., Martín-Pintado, J., Fuente, A., \& Wilson, T. L. 2004, A\&A, 427, 217

Rolleston, W. R. J., Smartt, S. J., Dufton, P. L., \& Ryans, R. S. I. 2000, A\&A, 363,537

Roy, S. 2013, ApJ, 773, 67

Samson, J. A. R., \& Angel, G. C. 1990, Phys. Rev. A, 42, 1307

Sofia, U. J., Lauroesch, J. T., Meyer, D. M., \& Cartledge, S. I. B. 2004, ApJ, 605, 272

Steigman, G., Werner, M. W., \& Geldon, F. M. 1971, ApJ, 168, 373

Torii, K., Kudo, N., Fujishita, M., et al. 2010, PASJ, 62, 1307

Velusamy, T., \& Langer, W. D. 2014, A\&A, 572, A45

Velusamy, T., Langer, W. D., Pineda, J. L., \& Goldsmith, P. F. 2012, A\&A, 541, L10

Verner, D. A., Yakovlev, D. G., Band, I. M., \& Trzhaskovskaya, M. B. 1993, Atom. Data Nucl. Data Tables, 55, 233

Wang, Q. D., Gotthelf, E. V., \& Lang, C. C. 2002, Nature, 415, 148

Wenger, T. V., Bania, T. M., Balser, D. S., \& Anderson, L. D. 2013, ApJ, 764, 34

Wiesenfeld, L., \& Goldsmith, P. F. 2014, ApJ, 780, 183

Wilms, J., Allen, A., \& McCray, R. 2000, ApJ, 542, 914

Wolfire, M. G., Hollenbach, D., \& McKee, C. F. 2010, ApJ, 716, 1191

Young, E. T., Becklin, E. E., Marcum, P. M., et al. 2012, ApJ, 749, L17 